\title{
The host-associated archaeome
}

Guillaume Borrel ${ }^{1}$, Jean-François Brugère ${ }^{2}$, Simonetta Gribaldo ${ }^{1}$, Ruth A. Schmitz ${ }^{3}$ and Christine MoisslEichinger ${ }^{4,5 *}$

1 Evolutionary Biology of the Microbial Cell Unit, Department of Microbiology, Institut Pasteur, Paris, France.

2 Institut Universitaire de Technologie, Université Clermont Auvergne, CNRS, Laboratoire Microorganismes: Genome et Environnement - LMGE, Clermont-Ferrand, France.

3 Institute for General Microbiology, Christian Albrechts University of Kiel, Germany

4 Diagnostic and Research Institute of Hygiene, Microbiology and Environmental Medicine, Medical University Graz, Austria

5 BioTechMed, Graz, Austria

*email: christine.moissl-eichinger@medunigraz.at 


\section{Abstract}

Host-associated microbial communities have an important role in shaping the health and fitness of plants and animals. Most studies have focused on the bacterial, fungal or viral community, but have often neglected the archaeal component. The archaeal community, the so-called archaeome, is now growingly recognized as an important component of host-associated microbiomes. It is composed of various lineages, including mainly Methanobacteriales and Methanomassiliicoccales (Euryarchaeota), as well as representatives of the Thaumarchaeota. Host-archaeome interactions were mostly delineated from methanogenic archaea in the gastrointestinal tracts, where they contribute to substantial methane production, and are potentially also involved in disease-relevant processes. In this Review, we discuss the diversity and potential role of archaea associated with protists, plants and animals. We also present our current understanding of the archaeome in humans, the specific adaptations involved in interaction with the resident community as well as the host, and its role in health and disease. 


\section{Introduction}

Eukaryotes are inhabited by microorganisms, and there is increasing appreciation that this resident microbial community interacts with their host, and influences host fitness and functionality. These communities likely evolved over millions of years of coexistence with their hosts, leading to the holobiont concept ${ }^{1-4}$.

Most studies have focused on host-associated bacteria, but archaea have generally been neglected, despite the fact that they are also consistent members of microbiomes associated with diverse hosts, including protists, plants, animals and humans. Specifically, we know for almost half a century that methanogenic archaea thrive in the human gastrointestinal tract $(\mathrm{GIT})^{5}$, and the first representative, Methanobrevibacter smithii, was isolated nearly 40 years ago ${ }^{6}$.

Archaea were originally discovered and isolated from extreme ecosystems, including volcanic environments, salt lakes and other biotopes that are characterized by extreme temperatures, $\mathrm{pH}$ values or ion concentrations. However, over the past decades, cultivation-independent studies have revealed that archaea are universally distributed and could be among the most abundant and active microorganisms in moderate environments such as the ocean water column ${ }^{7-11}$. The domain Archaea includes a vast diversity of lineages, some of which are mostly composed of uncultured representatives ${ }^{12,13}$. These lineages are gathered into at least four large clades, namely the Euryarchaeota, the TACK (Thaumarchaeota, Aigarchaeota, Crenarchaeota, Korarchaeota) superphylum, the DPANN (Diapherotrites, Parvarchaeota, Aenigmarchaeota, Nanoarchaeota) clade and the Asgard archaea (Fig. 1). Currently known host-associated archaea are phylogenetically diverse, but are mostly composed of methanogens and, more recently, Thaumarchaeota (Fig. 1). Today, research on the human archaeome (defined as the archaeal component of the human-associated microbial community) is still in its infancy. This is due to various reasons, including methodological issues resulting from their specific biology (Box 1), which also leads to a general lack of knowledge about archaea in the microbiome research 
community. Recent studies provide new insights into the human archaeome, including, for example, the discovery of novel host-associated lineages, such as the Methanomassiliicoccales, which might have a beneficial impact on human health ${ }^{14,15}$, the discovery of archaea on the human skin and their possible link to age and skin physiology ${ }^{16,17}$, the finding that human archaea are recognized by the immune system and are involved in proinflammatory processes ${ }^{18,19}$. In addition, the development of specific archaea-targeting methods has updated the biogeography of the human archaeome and revealed previously undetected members ${ }^{20,21}$. Furthermore, novel insights were also gained into the role of archaea in plant physiology and the plant-specific profile of the archaeome ${ }^{22}$, their association with animal skin ${ }^{23}$ and the GIT of primates ${ }^{24}$, which has fueled the debate on archaeome host-adaptation and co-evolution.

With novel methods in place, even if still imperfectly adapted to the archaeome (Box 2), many fundamental questions about the contribution of archaea to the microbiome and host physiology can now be addressed (see Refs. ${ }^{25-36}$ ).

In this Review, we aim to provide a comprehensive view of the current knowledge on the host-associated archaeome, including humans, protists, plants and animals. In addition, we highlight the potential role of archaea in human health and disease, and explore the question of whether pathogenic archaea exist. Finally, we identify knowledge gaps that remain to be addressed and advise next steps for research on the archaeome.

\section{Protist, plant and animal archaeomes}

\section{The archaeome of anaerobic protists.}

Similarly to bacteria, archaea (namely members of the orders Methanobacteriales, Methanomicrobiales, Methanosarcinales and possibly Methanomassiliicoccales ${ }^{37}$ ) can live in the cytoplasm of anaerobic ciliates, 
amoebas and flagellates ${ }^{38}$. These endosymbiotic methanogens interact closely with hydrogenosomes, which are specific organelles of protists that generate $\mathrm{H}_{2}$ by oxidizing pyruvate and malate during carbohydrate degradation ${ }^{39}$. Methanogens benefit from the $\mathrm{H}_{2}$ for methanogenesis, and in return, they improve the energy gain of the host cell by maintaining a low $\mathrm{H}_{2}$ concentration ${ }^{40}$ (Box 1). In sediments and wetland soils, where free-living sulfate-reducing or iron-reducing bacteria are more competitive for $\mathrm{H}_{2}$ than free-living methanogens, endosymbiotic methanogens can remain active by benefiting from an exclusive source of $\mathrm{H}_{2}$ produced by hydrogenosomes. In such contexts, endosymbiotic methanogens can be responsible for the largest part of the methane production in these environments ${ }^{41,42}$.

Adding another order of complexity to the holobiont concept, methanogenic archaea can also be endosymbionts (and ectosymbionts ${ }^{43}$ ) of protists living inside the gut of termites ${ }^{44}$, cockroaches ${ }^{45}$, amphibians ${ }^{46}$ and ruminants (reviewed in Refs. ${ }^{47,48}$ ), where they have a role in host digestion together with the rest of the microbiota ${ }^{49}$ (Fig. 2). In addition to the $\mathrm{H}_{2}$ produced by their host cell, endosymbiotic methanogens also benefit from $\mathrm{O}_{2}$ depletion by protists, as most methanogens are extremely oxygen sensitive organisms (Box 1), during ruminant feeding ${ }^{50}$. In support of this assumption, methane emissions from ruminants are positively correlated with protist concentrations ${ }^{51}$, and defaunation experiments (that is, removal of protists) led on average to an $11 \%$ reduction of methane emissions, albeit with a great variability between studies (from no effect up to $37 \%$ reduction ${ }^{47,52}$ ). Thus, this variability suggests that the relationships between methane emissions and protists also include complex and not well understood factors ${ }^{53,54}$.

Whether endosymbiotic archaea form stable association with their host or are the result of random engulfment of free-living archaea by the protist has been debated ${ }^{37,55,56}$. On one hand, archaea-protist associations are not maintained on long evolutionarily periods as shown by the absence of co-speciation patterns between the host and the archaea ${ }^{46}$. On the other hand, specific associations are supported by the observations that geographically distant representatives of the same protist species host similar methanogens, 
whereas different protist species in the same location host different methanogens ${ }^{38,57,58}$. Moreover, recent genomic analyses of endosymbiotic archaea have revealed only little differences with their free-living counterparts 59,60 , but the nature of these variations seems partly similar between evolutionary distant endosymbiotic archaea, which suggests convergent adaptations to this lifestyle ${ }^{58}$. In addition, the high level of genes undergoing pseudogenization in these genomes probably indicates a recent and still ongoing process of adaptation ${ }^{58}$. Together, these studies suggest that endosymbiotic archaea form stable associations with their host, probably at the strain level, but are periodically replaced by a novel archaeal symbiont. However, most of these observations derive from associations between archaea and free-living protists, and the question still remains open for host-associated protists. 


\section{The plant archaeome.}

Microbial communities of plants have an essential role as they can affect plant growth, productivity, adaptation, diversification and health ${ }^{61}$. Overall, archaea are differently distributed in the rhizosphere , endosphere and phyllosphere. Micro-niche differentiation is supported by the competition with bacteria and fungi as well as abiotic factors, including nutrient availability and exposure in the phyllosphere, the presence of root exudates in the rhizosphere, and the more stable conditions in the soil ${ }^{62}$. For the widespread leafy green plant arugula (rocket salad), the diversity of archaea was found to be lowest in the phyllosphere, which indicates unfavorable habitat conditions, whereas the diversity of archaea in the soil and rhizosphere was much richer, and may thus be the preferred habitat ${ }^{62}$.

However, knowledge on the interaction of archaea and plants is very restricted and based on a few specific types of plant-archaeome interactions. The most prominent example of plant-associated archaea are methanogens residing in the anaerobic rhizosphere of rice in oxygen-depleted wetlands ${ }^{63}$, mostly represented by Methanocellales, Methanosaetaceae and Methanoregulaceae ${ }^{64}$. A large part of the methane produced in the rice rhizosphere is derived from the breakdown of organic compounds produced by the plant ${ }^{63,65}$, and it primarily escapes to the atmosphere via the plant gas vascular system, thus bypassing bacterial aerobic methanotrophs ${ }^{66}$. Although methane emission in rice fields is a subset of the overall plant-mediated methane emission in wetlands, it is responsible for $10 \%$ of the global budget of atmospheric methane ${ }^{67}$.

Signatures of ammonia oxidizing Thaumarchaeota were also found to be abundant in leaves of Mediterranean olive trees ${ }^{68}$, which reveals cultivar-specific abundance patterns. Similar observations were made in tomato plants, where the abundance of the archaeal community (Thaumarchaeota (60\%), Methanosarcina (12.6\%), Methanoculleus (3.4\%); Fig. 3) was found to be dependent on plant genotype and habitat. Notably, the archaeal abundance and diversity was comparably low in seeds, and no indications of plant-mediated vertical transmission of archaeal microbiome components was detected ${ }^{69}$. 
Plants in alpine bogs harbor a substantial archaeal community that is composed of 60 different genera ${ }^{22}$. Notably, metagenomic analyses revealed potential archaeal functions in, for example, the promotion of plant growth through auxin biosynthesis, nutrient supply, and protection against oxidative and osmotic stress. Additional genetic capacities for $\mathrm{CO}_{2}$ and $\mathrm{N}_{2}$ fixation were also observed. Similar functions were reported for the arugula, with mainly Thaumarchaeota and Euryarchaeota detectable and visible in both the rhizosphere and phyllosphere ${ }^{62}$. In particular, 'Candidatus Nitrosocosmicus' (Thaumarchaeota) seems to be involved in positive interaction with plants, as Nitrosocosmicus oleophilus MY3 was found to colonize root surfaces of Arabidopsis thaliana plants and to trigger systemic resistance against the plant pathogens Pectobacterium carotovorum subsp. carotovorum SCC1 and Pseudomonas synringae pv. tomato DC3000 ${ }^{70}$.

\section{The animal archaeome.}

Known symbiotic associations of archaea with animals include sponges, insects and vertebrates. The first representative genome of Thaumarchaeota, namely 'Candidatus Cenarchaeum symbiosum', was retrieved from a marine sponge, which lives in close symbiosis with its archaeal inhabitant ${ }^{71,72}$. In some cases, Thaumarchaeota even dominate the microbial communities associated with sponges ${ }^{73}$. It was suggested that these archaea might remove nitrogenous host-waste products and, in turn, provide carbon to the host ${ }^{71}$.

Methanogenic archaea, and in particular Methanobrevibacter species, are extraordinarily well-adapted to interact with animal hosts and non-archaeal components of their microbiomes. By their consumption of various small fermentation end-products, Methanobrevibacter species are flexible supporters of syntrophic interactions. Methanobrevibacter species are the predominant archaea in gastrointestinal tracts (GIT) of various ruminants and non-ruminants, such as cattle, yak, sheep, reindeer, goat, buffalo, deer, pigs, wallabies, rhinos, chicken, iguanas, termites and many others (see Ref. ${ }^{25}$ ) (Figs. 2,3). Other methanogenic archaea (Methanosphaera, Methanosarcina, Methanomassiliicoccus and Methanimicrococcus species) have also been 
identified in various animals (for example, cattle, sheep, goat, deer, horses, pigs, kangaroos, rhinoceros, hoatzin, iguana and termites), but they are usually less abundant (Fig. 4). Due to the resulting massive methane production and impact on global warming (Fig. 2), methanogenic archaea-ruminant and archaeatermite symbiosis is the subject of active research. It has been estimated that a single cow emits up to $700 \mathrm{~L}$ of methane per day ${ }^{74}$ (on average $150.7 \mathrm{~g}$ /day; see Ref. ${ }^{25}$ ), and these studies also aim to reduce methane emission ${ }^{75}$. In addition, the activity of methanogenic archaea negatively affects the weight gain and efficiency of feeding, so that a reduction of the methanogenic archaea load (generally $\sim 4 \%$ ) in the rumen is a sought outcome ${ }^{76}$. Also, the termite archaeome is a substantial contributor to biotic methane emission. It is estimated that approximately $20 \mathrm{Tg}$ (Teragram) of methane are produced by those insects globally each year ${ }^{77}$, but the overall contribution is considered rather low (1\%-3 \% of total methane budget). This discrepancy is explained by the observation that $20 \%-80 \%$ of the termite-produced methane is depleted by bacterial methanotrophs which reside in the mound or soil in close proximity to the termites ${ }^{77}$.

A recent study assessed archaeal diversity in great apes faecal samples, which detected more than 200 archaeal taxa, with the highest diversity observed for orangutans ${ }^{24}$. More specifically, Methanobacteriales, Methanomassiliicoccales and Thaumarchaeota were all detected in the GIT of the analyzed primates. Notably, it was proposed that the diversification of great apes correlated with a decline of archaeal diversity in the GIT ${ }^{24}$, which potentially indicates a diet-associated loss of archaeal taxa during primate evolution, and improved fitness of remaining taxa.

Besides archaeal communities in the GIT, various animals also carry methanogens, Haloarchaea and Thaumarchaeota on their skin (Fig. 3). The relative abundance of skin archaea was found to be speciesspecific $^{23}$ and reached up to $26 \%$ of archaeal sequence proportions (averaged) in cape elands (Fig. 3). However, the overall archaeal proportion in this study was $\sim 0.1 \%$ of all retrieved sequences, even though a non-archaea-specific approach was used, and thus the archaeal load might have been underestimated. 
Interestingly, and although the functional capability of the detected archaea is largely unknown, the variety of skin-associated archaea in mammals was very similar to the skin archaeome found in humans (see below; Fig. 3).

Current available information suggests that only specific archaeal clades are prone to interaction with hosts and non-archaeal components of their microbiomes (Figs. 3, 4). Thaumarchaeota (also referred to as Nitrososphaeria; with many unclassified representatives) are mainly associated with the host outer or exposed surfaces (such as, human and animal skin, or the plant's phyllosphere), whereas methanogenic archaea (Methanobacteriales, Methanosarcinales, Methanomicrobiales, Methanomassiliicoccales) are frequently found in anoxic areas (mostly the GIT of animals and humans). Both archaeal groups consume metabolic endproducts of the host and the associated bacteriome, and exhibit specific functions which can protect or negatively affect the host.

In addition, the consistent detection of halophilic archaea (particularly Halococcus; Fig. 4) in animals (and plants) raises many questions on the origin and the type of interaction ${ }^{27}$, particularly as it was discussed for humans that halophilic archaea could be contaminants from salted food ${ }^{78}$.

This is also true for the unclassified 'Candidatus Woesearchaeota' (DPANN) which have also been identified in human samples, mainly from the respiratory tract ${ }^{20}$. The function of Woesearchaeota in the environment remains elusive, but due to the metabolic deficiencies, a probable dependency on syntrophic microorganisms has been discussed ${ }^{79}$; details on their potential role in the human body are completely unknown to date.

\section{The human archaeome}

The human microbiome carries numerous archaea, in particular on skin, in the respiratory tract and the GIT. Whereas the specific role of non-methanogenic archaea in the human body remains to be explored, 
methanogens maintain numerous syntrophic relationships with the resident bacteria. Due to their dependence on bacterial metabolic activity for their own substrate availability, methanogens could be indicators for the

microbiome status per se ${ }^{32,80,81}$. Despite limited knowledge on host interactions, archaeal genomes and experiments with cultured methanogen representatives reveal a profound adaptation strategy to the human GIT.

\section{Presence, abundance and activity of archaea in the human microbiome.}

Archaea are substantial components of the human microbiome and include a wide diversity of lineages, including Methanobacteriales, Methanomassiliicoccales, Methanomicrobiales, Methanosarcinales, Halobacteriales, Thaumarchaeota (Nitrososphaeria), and members of the DPANN clade ${ }^{15,20,35}$ (Figs. 1, 3).

In the GIT, the most prevalent and abundant archaea are representatives of the Methanobacteriales and the Methanomassiliicoccales ${ }^{15}$. Methanobacteriales are mainly represented by two species, namely Methanobrevibacter smithii and Methanosphaera stadtmanae, having a prevalence of up to $97.5 \%$ and $23 \%$, respectively ${ }^{82}$. Human gut-associated Methanomassiliicoccales consist of at least nine species, the most common being 'Candidatus Methanomassiliicoccus intestinalis', 'Candidatus Methanomethylophilus alvus', $\mathrm{Mx}-02, \mathrm{Mx}-03$ and $\mathrm{Mx}-06$, with a prevalence of up to $80 \%{ }^{15}$. Together, these methanogens contribute to an average human body methane emission of about $0.35 \mathrm{I}$ per day ${ }^{83}$. Considering a world population of 7.5 billion people, the total human methane emission would be equivalent to $410 \pm 113 \mathrm{Gg}$ per year (Fig. 2). Although this seems to be a high amount, it represents less than $0.2 \%$ of the overall anthropogenic emitted methane ${ }^{84}$.

Several factors influence the presence, abundance and diversity of archaea in the human gut. For example, the percentage of people emitting breath methane above $1 \mathrm{ppm}$ correlates with geography and ethnicity, which indicates an influence of host genetics and life history, gut microbiota composition and diet. Of note, the 
methods used to measure breath methane have been frequently debated ${ }^{85}$, as inconsistent values can be obtained based on test methodology (for example, prior subject challenge with carbohydrates), selected cutoff values, and correction of raw measurements or interpretation. As summarized in a recent publication ${ }^{83}$, only $15 \%$ of the Japanese population ${ }^{86}$ but more than $70 \%$ of rural Africans ${ }^{87,88}$ were shown to emit methane levels above the $1 \mathrm{ppm}$ threshold. Interestingly, compared to native Africans, African Americans showed statistically significant lower methane emission and methanogen diversity ${ }^{89,90}$, which suggests a negative effect of western diet or lifestyle on the GIT archaeome.

In Western adult populations, around $40 \%-60 \%$ of individuals were found positive following the methane breath test (with a threshold of $1 \mathrm{ppm})^{91}$ and thus are estimated to carry more than $10^{7}-10^{8}$ methanogens per gram of stool 92,93 , which corresponds to a minimum of $0.03 \%-0.3 \%$ of the gut microbiota. However, the proportion of methanogens can greatly vary above this level and reach up to $14 \%$ of the gut microbiota in extreme cases, as found in a Russian cohort via untargeted metagenomic analyses ${ }^{81}$.

Another important factor is age, correlating positively with the diversity and abundance of gut-associated archaea in several human populations ${ }^{83,94}$. Whether this increase is due to host physiological changes, such as the prolonged gastrointestinal transit time with age, and/or multiple acquisitions during human life, remains unclear. Another factor is host genetics, as the abundance of $M$. smithii has been found to be more similar between monozygotic twins than between dizygotic twins ${ }^{95-97}$, and correlates positively with a SNP in a long noncoding RNA of the human genome ${ }^{98}$. However, the presence and activity of methanogens is also associated with non-archaeal members of the host microbiome. For instance, the prevalence of Methanomassiliicoccales, which reduce trimethylamine (TMA) with $H_{2}$ for methanogenesis, was found to correlate positively with the number of different TMA-producing pathways present in the bacterial microbiome ${ }^{15}$. Similarly, a correlation of $M$. smithii with certain bacterial taxa from the Firmicutes was noted. In particular, Christensenellaceae, representing a highly heritable clade ${ }^{99,100}$ were found to be associated with 
a high abundance of Methanobrevibacter. Christensenella representatives support M. smithii through efficient $\mathrm{H}_{2}$ transfer via close physical interactions, as shown in co-culture experiments with $C$. minuta ${ }^{100}$. In these experiments, the hydrogen consumption by $M$. smithii shifted the $C$. minuta metabolism towards acetate production rather than butyrate production, an effect that was less pronounced with Bacteroides thetaiotaomicron, a taxon not correlating with M. smithii abundance in the human gut. Interestingly, nonbacterial microbiome members such as Candida fungi were also found to co-occur with $M$. smithii, which suggests additional syntrophic relationships ${ }^{101}$.

Besides methanogens, Halobacteriales (for example, Haloferax massiliense) have been detected and isolated from human stool samples, including from patients suffering from inflammatory bowel disease (IBS) ${ }^{35,78,102,103}$. However, the impact of halophilic archaea on the human microbiome and the host remains unclear, and their presence has been discussed as possibly transient and associated with consumption of salt-containing food products ${ }^{104}$

The overwhelming majority of studies on human-associated archaea were conducted on stool samples, and knowledge on specific body sites is still sparse. It seems likely that the GIT contains a larger diversity of archaea than that identified from stool samples, as signatures of Methanomicrobiales, Methanobacterium and DPANN were reported in biopsy samples but not in the stool ${ }^{20,90}$. Moreover, similar to bacteria, human-associated archaeal communities group based on the body location, with Thaumarchaeota signatures predominating on the skin, methanogens in the GIT, a mixed Thaumarchaeota or methanogens landscape for the upper respiratory tract, and DPANN (Woesearchaeota) in the lung ${ }^{20}$. On the skin, two studies revealed that archaea generally represent $0.1 \%$ to $1 \%$ of the microbiota ${ }^{16,17}$. Interestingly, the positive correlation between age and abundance of archaea observed for the gut also holds true for the skin ${ }^{17}$. 


\section{Adaptations to the human host and interaction with the immune system.}

As discussed above, archaea in the human gut are generally dominated by a few specific taxa (Fig. 3). These taxa are rarely reported from environments outside of the animal GIT, which suggests a high degree of specialized adaptation. Such adaptations are mirrored by a number of specific traits differentiating archaea residing in the GIT from free-living ones. These traits include, for example, modifications of the cell surface (for improved adhesion and biofilm formation), and the possession of bile salt hydrolases, to defeat the host defense mechanisms (Fig. 5).

M. smithii, M. stadtmanae and host-associated Methanomassiliicoccales encode a large number of membrane-bound adhesin-like proteins (ALPs) that have been suggested to be involved in binding to different host sites and syntrophic commensal bacteria ${ }^{15,105-107}$. As the expression levels of $M$. smithii ALPs are influenced by environmental conditions (for example, the presence of bacteria or substrate availability), it has been suggested that $M$. smithii has a high ability to colonize different microniches in the gut ${ }^{96,105}$. It could be hypothesized that the specific physical interaction of $M$. smithii with $C$. minuta ${ }^{100}$ is promoted by some of these ALPs. Methanobacteriales and Methanomassiliicoccales ALPs comprise different protein domains ${ }^{15,96}$, possibly indicating different niche adaptations and evolutionary origins. Phylogenetic approaches revealed that several of the M. smithii ALPs have been probably acquired by horizontal gene transfer (HGT) ${ }^{96,108}$. Methanomassiliicoccales have probably also acquired their ALPs via HGT, as the type they possess has not been found in other archaea but is present in high numbers in several gut-associated bacteria ${ }^{15}$.

Besides ALPs, diverse glycosyltransferases seem to have been acquired via HGT in Methanobrevibacter and Methanosphaera species. These glycosyltransferases could account for a modification of polysaccharides present at the cell surface, which could improve adherence to abiotic and biotic surfaces ${ }^{105,107,108}$. Supporting this observation, M. stadtmanae cells strongly adhere to human immune and epithelial cells ${ }^{109}$, and easily 
aggregate into biofilm structures, most probably due to secretion of extracellular polysaccharides ${ }^{109}$. Incidentally, oral biofilms were reported to contain Methanobrevibacter oralis (in at least every second patient suffering from periodontal disease ${ }^{110}$ ), and members of the Methanomassiliicoccus genus ${ }^{111}$.

Bile acids are important regulators of the human microbiome and exert a strong selective pressure on the microbial population. Microorganisms have developed strategies to counteract bile toxicity via bile salt hydrolases (BSHs) which are also important for secondary bile acid synthesis. Similar to various bacteria, several gut methanogens, M. smithii, M. stadtmanae and Ca. M. alvus, could detoxify this molecule using BSH. Methanobacteriales and Methanomassiliicoccales BSH are distantly related and could have been acquired via independent HGT events ${ }^{112}$, probably from Firmicutes in the case of Methanobacteriales ${ }^{113}$. $M$. oralis (mainly present in the mouth) and Methanomassiliicoccales from an environmental clade lack BSH ${ }^{35,112}$, an observation which supports the acquisition of this enzyme due to a very specific adaptation to the intestine.

The innate immune system is the very first line of host defense against microorganisms, including the production and release of antibacterial compounds such as antimicrobial peptides (AMP), along with cytokines. These are excreted by epithelial cells right after the recognition of microorganism-associated molecular patterns (MAMPs) of bacteria, such as flagellins, peptidoglycan and lipopolysaccharides (LPS). Although probably not directly the target, human archaea are exposed to the various AMPs secreted by the host to control the bacterial microbiota. Notably, susceptibilities against AMPs were found to be substantially different among mucosa-associated methanogenic strains ${ }^{19,114}$, with pseudomurein-containing archaea, such as M. stadtmanae, being more resistant against the lytic effects of AMPs than, for example, members of the Methanosarcinales or Methanomassiliicoccales.

In recent years it was clearly shown that archaea interact and activate the human immune system. Activation of human immune cells, as well as pro-inflammatory cytokine responses by peripheral blood mononuclear 
cells and by monocyte-derived dendritic cells, initiated by phagocytosis and endosomal lysis, was demonstrated ${ }^{18}$. Strong response, that is high release of pro-inflammatory cytokines including interleukins as well as interferons, was exclusively observed when stimulating with $M$. stadtmanae cells ${ }^{18,115}$. Interestingly, the other two GIT archaeal isolates tested, M. smithii and Methanomassiliicoccus luminyensis, showed only mild responses, if at all ${ }^{18,19}$. As $M$. smithii has the capacity to produce glycans that mimic those found in the human gut ${ }^{105}$, it is attractive to hypothesize that those host-like glycans enable M. smithii to escape from the host immune system. Although these studies demonstrated not only innate, but also adaptive immune response by human immune cells in response to $M$. stadtmanae, initially no specific receptor involved was identified. Only recently it was demonstrated that RNA of $M$. stadtmanae is a potent immune stimulator, and Toll-like receptor (TLR) 7 and TLR8 were identified as the involved pattern-recognition receptors, respectively

${ }^{116}$. Moreover, this molecular interaction led to TLR8-dependent triggering of the NLRP3 inflammasome in a new and alternative path of inflammasome activation. To the best of our knowledge, archaea do not trigger any other innate immune receptor and may thus be unique among microbial stimulators in triggering RNAdependent signaling only. Hence, this TLR8-dependent alternative inflammasome activation may be archaeaspecific.

\section{Archaea in human health and disease.}

By using the indirect detection of methanogenic activity via methane breath tests, possible correlations between the occurrence of methanogens in the human GIT and various diseases have been analyzed since the late $70 \mathrm{~s}^{117}$. Since then, the relationship of methanogen abundance (cultivation-, quantitative PCR (qPCR)- and next-generation sequencing (NGS)-based analyses) or breath methane content with disease has been assessed in various (gastrointestinal) diseases and physiological states of the host. These include colon cancer, diverticulosis, diabetes, obesity and anorexia, inflammatory bowel diseases and many others (summarized in 
Refs. ${ }^{28,35,118}$ ). However, due to the above mentioned methodological issues of bacteria-centric methods or other pitfalls (Box 2), available information is contradictory and the involvement of archaea in human health and disease remains often blurry ${ }^{31,35,118}$.

When they are subject to dysbiosis or infection, several sites of the human body are known to present higher prevalence and abundance of methanogenic archaea. For example, $M$. smithii was reported in the vagina only in patients suffering from vaginosis, but was absent in healthy individuals ${ }^{119,120}$. Moreover, M. smithii was found in individuals with muscle abscesses ${ }^{104}$, pneumonia ${ }^{121}$ and urinary tract infections ${ }^{122}$, and, together with $M$. oralis, in patients with refractory sinusitis ${ }^{123} . M$. oralis was also reported in brain abscesses ${ }^{111,124}$, and has been associated with periodontitis ${ }^{110,125}$ or peri-implantitis ${ }^{126}$. Interestingly, $M$. oralis is more prevalent and abundant in severe periodontitis, but was not detected in healthy sites adjacent to periodontal pockets and was no longer present after healing, which highlights the specific association of $M$. oralis with the inflamed site ${ }^{110}$. All these dysbioses and infections consist in a strong increase or de novo colonization by anaerobic fermentative bacteria. This shift to anaerobic fermentative bacteria is accompanied by an increase of archaea that can reach a high proportion of the whole microbial community, namely up to $\sim 25 \%$ in brain abscesses ${ }^{111,124}$ and $18 \%$ in severe periodontitis ${ }^{110}$. Although methanogens are never the only microorganisms present at these infected sites, they are likely to promote the outgrowth of fermentative bacteria involved in the inflammation by lowering $\mathrm{H}_{2}$ concentrations (Box 1). In fact, other hydrogen-consuming microorganisms such as sulfate-reducing bacteria show an increased abundance in severe periodontitis and could fulfill the same role as methanogens ${ }^{127}$. Thus, methanogenic archaea might participate in such polymicrobial diseases through syntrophic interactions, representing one component of a 'unit of pathogenicity' besides bacterial partners ${ }^{128,129}$. Interactions of methanogenic archaea with pathogenic bacteria could actually occur in various diseases and not be limited to syntrophic partnerships. Indeed, a recent genome survey revealed that more than 200 pathogens have genes involved in hydrogen consumption or production ${ }^{130}$. This suggests a potential 
dual role for methanogens, as syntrophic partners of fermentative pathogens and as potential competitors of the hydrogenotrophic ones.

Beyond this indirect role, it is unknown whether archaea can be directly involved in inflammation at these infected sites. Some indications exist for M. oralis, as it has only been detected in inflamed areas so far. In this respect, it has also been shown that the pro-inflammatory potential of archaea varies among species as, for example, $M$. stadtmanae triggers a stronger immune response than $M$. smithii and $M$. luminyensis using monocyte-derived dendritic cells ${ }^{29}$. Moreover, increased abundance of $M$. stadtmanae was found to frequently correlate with disease and inflammation, in particular in inflammatory bowel disease ${ }^{118,131}$. In combination with its observed high pro-inflammatory potential, activation of the inflammasome, as well as strong B-cell and T-cell responses within the draining lymph nodes due to $M$. stadtmanae entering the bloodstream ${ }^{18,115,132}$, suggest a potential involvement in the development and or manifestation of disease. By contrast, a recent publication showed an association between $M$. stadtmanae carriage and a lower risk of asthma in young children, which is indicative for a beneficial role for M. stadtmanae ${ }^{132}$.

Besides the direct interaction of archaeal cells and the immune system, the gaseous product of methanogenic archaea, methane, could have by itself a physiological effect on the host, as indicated by a recent growing number of studies. For example, a direct influence of methane on gut motility was shown, leading to a reduced faeces transit time by up to $59 \%$. This slow-down is possibly caused by a direct action of methane on the cholinergic pathway of the enteric nervous system ${ }^{133}$, possibly explaining also the association of methanogens with constipation (IBS-C type) ${ }^{134,135}$. Constipation, or longer faeces transit times, also ease the colonization of microorganisms with longer generation time, such as preferred syntrophic partners of methanogens (for example, Clostridiales cluster XIV) ${ }^{136}$, and methanogens themselves ${ }^{137}$. Treatment with statins, which specifically inhibit the archaeal fatty acid synthesis pathway, is considered as a possible way to improve constipation and associated disorders ${ }^{138}$. Other recent works, performed with methane-rich saline in rodent 
models, indicate that methane might be involved in other important processes ${ }^{139}$, such as enhanced exercise capacity ${ }^{140}$, increased secretion of GLP-1 (glucagon-like peptide-1, which has a role in insulin secretion and appetite suppression) ${ }^{141}$, or anti-inflammatory and neuroprotective effects ${ }^{142}$. Whether the methane formed by methanogens directly in the GIT also has these effects remains to be analyzed.

In addition to these potential positive effects of methane (which need to be re-evaluated in the human setting with biogenic methane supplied by methanogens), several other positive roles for archaea have been proposed. For example, Thaumarchaeota found on the skin could contribute to oxidation of ammonia compounds delivered by sweat, and thus lower the skin $\mathrm{pH}$. Their presence has in fact been linked with drier skin ${ }^{17}$, but details on beneficial, commensal or opportunistic pathogenic activities are still missing. Another important positive role of members of the Methanomassiliicoccales in human health could be through their utilization of TMA as a substrate for methanogenesis. TMA is generated during dietary compound degradation by intestinal bacteria and is then oxidized into trimethylamine-oxide (TMAO) in the liver ${ }^{143,144}$. TMAO is involved in the development of cardiovascular and chronic kidney diseases ${ }^{144,145}$, and TMA itself is associated with a genetic metabolic disorder, trimethylaminuria ${ }^{146}$. Methanomassiliicoccales species with the genetic potential to use TMA were found to be associated with a lower concentration of fecal TMA when compared to subjects without Methanomassiliicoccales or with Methanomassiliicoccales not capable of TMA consumption 15. The removal of TMA by Methanomassiliicoccales before it enters the bloodstream could therefore help prevent the development of diseases and metabolic disorders associated with this dietary nutrient.

The anaerobic TMA-degradation pathway, which requires the $22^{\text {nd }}$ proteinogenic amino acid pyrrolysine ${ }^{147-149}$, is shared by only a few bacteria and some archaea, and in the human GIT it currently seems to be unique to the Methanomassiliicoccales ${ }^{147-149}$. Therefore, the prevention of these diseases could rely on the supplementation of TMA-consuming Methanomassiliicoccales (so-called 'archaebiotics' ${ }^{14}$ ). As a proof-ofconcept, a single inoculation of $M$. luminyensis B10 (the so-far unique isolate of Methanomassiliicoccales ${ }^{150}$ ) 
significantly lowered the concentration of plasma TMAO in standard C57BL/6 laboratory mice through a 30 days experiment, despite a very poor colonization ${ }^{151,152}$. M. smithii and two TMA-using methanogenic archaea (non-human and environmental) also showed a protective effect on a mouse model prone to atherosclerosis 151.

\section{Do archaeal pathogens exist?}

Despite the above described possible involvement of methanogenic archaea in several polymicrobial diseases ${ }^{129}$, archaeal pathogens according to Koch's postulates and their per se pathogenicity have not been identified to date.

Nonetheless, in theory, archaea have all the preconditions to develop into pathogens: they are genetically and metabolically diverse, widespread in the environment, capable to engage in warfare with their close relatives by various anti-archaeal compounds (such as sulfolobicin ${ }^{153}$ ), have been interacting with different hosts for millions of years, and they are recognized by the host immune system ${ }^{30,31}$. Thus, it was proposed that the current lack of identified archaeal pathogens might simply reflect a lack of knowledge due to our current inability to correctly detect them in disease patterns ${ }^{30}$.

By contrast, it has also been proposed that no archaeal pathogens exist, and this may be due to various reasons. One hypothesis is that archaea use different cofactors than eukaryotes, and as such they have no inherent advantage in becoming pathogens to acquire resources from their target ${ }^{154}$. However, archaea could take advantage by acquiring other metabolites than vitamins ${ }^{155}$.

Another hypothesis for the absence of archaeal pathogens was attributed to the fact that they have unique viruses, and thus cannot acquire virulence factors from bacterial and eukaryotic viruses; besides, the abundance of archaeal lytic viruses may prevent the maintenance of virulence factors in the mobile genetic 
pool ${ }^{156}$. However, lysogenic or integrated viruses can also carry virulence factors, and current knowledge of the diversity and genetic pool of archaeal viruses is highly incomplete, covering only a few non host-associated lineages ${ }^{157}$. Even less information is available on the virome of host-associated archaea and its interplay with that of host-associated bacteria and eukaryotes.

Another prospect is that the emergence of pathogenesis is a rare event that occurred only in a few bacteria and eukaryotes, but never in archaea ${ }^{156}$. Amongst the very few bacterial pathogens (estimated less than $1 \%$ of all bacterial species ${ }^{158}$ ), pathogenic Gram-negative proteobacteria deliver virulence factors into their target cells by using specialized molecular needles such as type III secretion systems, which are absent from archaea. However, many Gram-positive pathogens exist, and they deliver their virulence factors by other machineries that can also be found in the archaea, and so there is de facto no reason why an archaeon could not have acquired the capability to use one of these systems to deliver virulence factors to a eukaryotic cell.

As discussed in previous chapters, only a few lineages of the archaea have engaged the association with eukaryotic hosts (Fig.1). This small number of transitions from a free-living to a host-associate lifestyle in archaea may have narrowed the chances to develop virulence in a certain time frame, in particular when considering that a minimum of 42 independent events of adaptations to the host-associated lifestyle occurred in bacteria ${ }^{159}$.

This opens another question: why would archaea be inherently less capable than bacteria to adapt to a hostassociated life? The answer may be linked to a common ecological trait of the Archaea as proposed previously ${ }^{160}$ : the adaptation to chronic energetic stress. This trait relies primarily on the specific membranes of archaea that enables them to dominate in extreme environments by minimizing their maintenance energy and outcompete or be as competitive as bacteria in niches with low available energy. According to this hypothesis, 
the inherent tendency of archaea to thrive under chronic energy stress would therefore be incompatible with the rapid adaptability that is a distinguished feature of many pathogens ${ }^{160}$. Moreover, this ancestral trait may have restricted the environmental distribution of many archaea to specific niches, such as extreme environments and/or deep anoxic sediments, where they are unlikely to encounter a potential eukaryotic host.

In conclusion, it is striking that nearly 40 years after the first description of human-associated archaea, we still have no evidence for one of their members being the primary cause of a disease. The reasons for this are still unclear and probably lie in a combination of all the above-mentioned hypotheses, including the fact that the archaea that are the most strongly associated with the animal host, notably methanogens, depend indeed on the availability of metabolites $\left(\mathrm{H}_{2}\right.$, methyl compounds) produced by other resident bacteria, and as such, may be unable to act as independent pathogens.

\section{Conclusions and outlook}

Summarizing the current knowledge, it is evident that archaea are abundant, diverse and active components of numerous microbiomes in plants, animals, and humans. The current body of literature indicates that their presence has a substantial influence on their hosts and other members of the microbiome. However, it is still unclear whether these interactions rely on archaea-specific traits or properties shared with and/or acquired from other microbiome components. Either way, such adaptations are likely to be the result of a long-term coevolution. From a host perspective, and beyond the enigma of the absence of pathogenic archaea, the extent to which their interactions are beneficial, neutral or deleterious is still unknown. Moreover, no data are available on the intra-species and interspecies level communication between archaea and both their 
syntrophic partners and hosts. Open questions are also how and when archaea are acquired during the lifetime of their hosts, and why their diversity and abundance seem to increase with age. These questions are central but still unanswered due to methodological limitations and complex confounding factors affecting archaeal distribution (for example, host age or ethnicity). More efforts are therefore required to characterize and capture the diversity of host-associated archaea by using targeted approaches on well-characterized populations. There is also an urgent need for growing, isolating and characterizing a higher number of hostassociated archaeal strains, which will provide key knowledge on their physiology, besides that inferred from genome or metagenome sequences alone. This would also reveal the determinants that enabled certain archaea to successfully colonize their host. Finally, it will be important to study archaea-host associations through the development of plant and animal models with defined microbiomes. In addition to the abovementioned perspectives, future research on human health will need to include more clinical studies, and take into consideration the inflammation potential of archaea and their interaction with the immune system, especially of archaea that are strongly associated with inflamed body sites. These studies should also address the role of archaeal metabolisms and their products, such as the influence of methane on the human body.

\section{References}

1. Rosenberg, E., Koren, O., Reshef, L., Efrony, R. \& Zilber-Rosenberg, I. The role of microorganisms in coral health, disease and evolution. Nat. Rev. Microbiol. 5, 355 (2007).

2. Zilber-Rosenberg, I. \& Rosenberg, E. Role of microorganisms in the evolution of animals and plants: the hologenome theory of evolution. FEMS Microbiol. Rev. 32, 723-735 (2008).

3. McFall-Ngai, M. et al. Animals in a bacterial world, a new imperative for the life sciences. Proc. Natl. Acad. Sci. 110, 3229-3236 (2013). 
4. Theis, K. R. et al. Getting the hologenome concept right: an eco-evolutionary framework for hosts and their microbiomes. Msystems 1, e00028-16 (2016).

5. Calloway, D. H., Colasito, D. J. \& Mathews, R. D. Gases produced by human intestinal microflora. Nature 212, 1238-1239 (1966).

6. Miller, T. L., Wolin, M. J., de Macario, E. C. \& Macario, A. J. Isolation of Methanobrevibacter smithii from human feces. Appl. Environ. Microbiol. 43, 227-232 (1982).

First isolation of an archaeon from the human gastrointestinal tract.

7. DeLong, E. F. Archaea in coastal marine environments. Proc. Natl. Acad. Sci. 89, 5685-5689 (1992).

8. Schleper, C., Jurgens, G. \& Jonuscheit, M. Genomic studies of uncultivated archaea. Nat. Rev. Microbiol. 3, 479 (2005).

9. Auguet, J.-C., Barberan, A. \& Casamayor, E. O. Global ecological patterns in uncultured Archaea. ISME J. 4, 182 (2010).

10. Pereira, O., Hochart, C., Auguet, J. C., Debroas, D. \& Galand, P. E. Genomic ecology of Marine Group II, the most common marine planktonic Archaea across the surface ocean. Microbiologyopen 8, e00852 (2019).

11. Santoro, A. E., Richter, R. A. \& Dupont, C. L. Planktonic Marine Archaea. Ann. Rev. Mar. Sci. 11, 131-158 (2019).

12. Adam, P. S., Borrel, G., Brochier-Armanet, C. \& Gribaldo, S. The growing tree of Archaea: new perspectives on their diversity, evolution and ecology. ISME J. 11, 2407 (2017).

13. Spang, A., Caceres, E. F. \& Ettema, T. J. G. Genomic exploration of the diversity, ecology, and evolution of the archaeal domain of life. Science (80-. ). 357, eaaf3883 (2017).

14. Brugère, J. F. et al. Archaebiotics: Proposed therapeutic use of archaea to prevent trimethylaminuria and cardiovascular disease. Gut Microbes 5, (2013).

First proposition to provide archaea as live biotherapeutic products to prevent some human diseases

15. Borrel, G. et al. Genomics and metagenomics of trimethylamine-utilizing Archaea in the human gut microbiome. ISME J. (2017).

(Meta)genomic and metabolic diversity of human gut methanogens in a European elderly cohort, with evidence of lower fecal TMA concentration associated with some specific methanogens.

16. Probst, A. J., Auerbach, A. K. \& Moissl-Eichinger, C. Archaea on Human Skin. PLoS One 8, (2013).

17. Moissl-Eichinger, C. et al. Human age and skin physiology shape diversity and abundance of Archaea on skin. Sci. Rep. 7 (1), 4039 (2017).

18. Bang, C., Weidenbach, K., Gutsmann, T., Heine, H. \& Schmitz, R. A. The intestinal archaea Methanosphaera stadtmanae and Methanobrevibacter smithii activate human dendritic cells. PLoS One 9, e99411 (2014).

First demonstration of immunogenic activity of human-associated methanogens (severe proinflammatory response of peripheral blood mononuclear cells).

19. Bang, C., Vierbuchen, T., Gutsmann, T., Heine, H. \& Schmitz, R. A. Immunogenic properties of the human gut-associated archaeon Methanomassiliicoccus luminyensis and its susceptibility to antimicrobial peptides. PLoS One 12, e0185919 (2017).

20. Koskinen, K. et al. First insights into the diverse human archaeome: specific detection of archaea in the gastrointestinal tract, lung, and nose and on skin. MBio e00824-17 (2017).

NGS-based analysis of the archaeome at different body sites, revealing a specific biogeographic pattern.

21. Pausan, M. R. et al. Exploring the Archaeome: Detection of Archaeal Signatures in the Human Body. Front. Microbiol. 10, 2796 (2019). 
22. Taffner, J. et al. What Is the Role of Archaea in Plants? New Insights from the Vegetation of Alpine Bogs. mSphere 3, e00122-18 (2018).

Dedicated analyses of the bog plant archaeome.

23. Ross, A. A., Müller, K. M., Weese, J. S. \& Neufeld, J. D. Comprehensive skin microbiome analysis reveals the uniqueness of human skin and evidence for phylosymbiosis within the class Mammalia. Proc. Natl. Acad. Sci. 115, E5786-E5795 (2018).

24. Raymann, K., Moeller, A. H., Goodman, A. L. \& Ochman, H. Unexplored archaeal diversity in the great ape gut microbiome. mSphere 2, e00026-17 (2017).

25. Moissl-Eichinger, C. et al. Archaea Are Interactive Components of Complex Microbiomes. Trends Microbiol. 26, (2018).

26. Bang, C. \& Schmitz, R. A. Archaea: forgotten players in the microbiome. Emerg. Top. Life Sci. ETLS20180035 (2018).

27. de Macario, E. C. \& Macario, A. J. L. in (Endo) symbiotic Methanogenic Archaea 103-119 (Springer, 2018).

28. Chaudhary, P. P., Conway, P. L. \& Schlundt, J. Methanogens in humans: potentially beneficial or harmful for health. Appl. Microbiol. Biotechnol. 102, 3095-3104 (2018).

29. Bang, C. \& Schmitz, R. A. Archaea associated with human surfaces: not to be underestimated. FEMS Microbiol. Rev. 39, 631-648 (2015).

30. Cavicchioli, R., Curmi, P. M. G., Saunders, N. \& Thomas, T. Pathogenic archaea: do they exist? BioEssays 25, 1119-1128 (2003).

31. Eckburg, P. B., Lepp, P. W. \& Relman, D. A. Archaea and their potential role in human disease. Infect. Immun. 71, 591-596 (2003).

32. Horz, H.-P. \& Conrads, G. The discussion goes on: What is the role of Euryarchaeota in humans? Archaea 2010, 967271 (2010).

33. Aminov, R. I. Role of archaea in human disease. Front. Cell. Infect. Microbiol. 3, 42 (2013).

34. Dridi, B., Raoult, D. \& Drancourt, M. Archaea as emerging organisms in complex human microbiomes. Anaerobe 17, 56-63 (2011).

35. Gaci, N., Borrel, G., Tottey, W., O'Toole, P. W. \& Brugère, J.-F. Archaea and the human gut: New beginning of an old story. World J. Gastroenterol. WJG 20, 16062 (2014).

36. Saengkerdsub, S. \& Ricke, S. C. Ecology and characteristics of methanogenic archaea in animals and humans. Crit. Rev. Microbiol. 40, 97-116 (2014).

37. Levy, B. \& Jami, E. Exploring the Prokaryotic Community Associated With the Rumen Ciliate Protozoa Population. Front. Microbiol. 9, 2526 (2018).

38. Hackstein, J. H. P. (Endo) symbiotic methanogenic archaea. 19, (Springer, 2018).

39. Muller, M. The hydrogenosome. J. Gen. Microbiol. 139, 2879-2889 (1993).

40. Fenchel, T. O. M. \& Finlay, B. J. Endosymbiotic methanogenic bacteria in anaerobic ciliates: significance for the growth efficiency of the host. J. Protozool. 38, 18-22 (1991).

41. Holmes, D. E. et al. Methane production from protozoan endosymbionts following stimulation of microbial metabolism within subsurface sediments. Front. Microbiol. 5, 366 (2014).

42. Fenchel, T. Methanogenesis in marine shallow water sediments: The quantitative role of anaerobic protozoa with endosymbiotic methanogenic bacteria. Ophelia 37, 67-82 (1993).

43. Vogels, G. D., Hoppe, W. F. \& Stumm, C. K. Association of methanogenic bacteria with rumen ciliates. Appl. Environ. Microbiol. 40, 608-612 (1980).

44. Lee, M. J., Schreurs, P. J., Messer, A. C. \& Zinder, S. H. Association of methanogenic bacteria with flagellated protozoa from a termite hindgut. Curr. Microbiol. 15, 337-341 (1987). 
45. Gijzen, H. J., Broers, C. A., Barughare, M. \& Stumm, C. K. Methanogenic bacteria as endosymbionts of the ciliate Nyctotherus ovalis in the cockroach hindgut. Appl. Environ. Microbiol. 57, 1630-1634 (1991).

46. van Hoek, A. H. et al. Multiple acquisition of methanogenic archaeal symbionts by anaerobic ciliates. Mol. Biol. Evol. 17, 251-258 (2000).

47. Newbold, C. J., de la Fuente, G., Belanche, A., Ramos-Morales, E. \& McEwan, N. R. The Role of Ciliate Protozoa in the Rumen. Front. Microbiol. 6, 1313 (2015).

48. Patra, A., Park, T., Kim, M. \& Yu, Z. Rumen methanogens and mitigation of methane emission by antimethanogenic compounds and substances. J. Anim. Sci. Biotechnol. 8, 13 (2017).

49. Ushida, K. in (Endo) symbiotic Methanogenic Archaea 25-35 (Springer, 2018).

50. Lloyd, D., Hillman, K., Yarlett, N. \& Williams, A. G. Hydrogen production by rumen holotrich protozoa: effects of oxygen and implications for metabolic control by in situ conditions. J. Protozool. 36, 205-213 (1989).

51. Guyader, J. et al. Influence of rumen protozoa on methane emission in ruminants: a meta-analysis approach. Animal 8, 1816-1825 (2014).

52. Hegarty, R. S. Reducing rumen methane emissions through elimination of rumen protozoa. Aust. J. Agric. Res. 50, 1321-1328 (1999).

53. Morgavi, D. P., Forano, E., Martin, C. \& Newbold, C. J. Microbial ecosystem and methanogenesis in ruminants. Animal 4, 1024-1036 (2010).

54. Morgavi, D. P., Martin, C., Jouany, J.-P. \& Ranilla, M. J. Rumen protozoa and methanogenesis: not a simple cause-effect relationship. Br. J. Nutr. 107, 388-397 (2012).

55. Park, T. \& Yu, Z. Do Ruminal Ciliates Select Their Preys and Prokaryotic Symbionts? Front. Microbiol. 9, 1710 (2018).

56. Henderson, G. et al. Rumen microbial community composition varies with diet and host, but a core microbiome is found across a wide geographical range. Sci. Rep. 5, 14567 (2015).

57. Lewis, W. H., Sendra, K. M., Embley, T. M. \& Esteban, G. F. Morphology and Phylogeny of a New Species of Anaerobic Ciliate, Trimyema finlayi n. sp., with Endosymbiotic Methanogens. Front. Microbiol. 9, 140 (2018).

58. Lind, A. E. et al. Genomes of two archaeal endosymbionts show convergent adaptations to an intracellular lifestyle. ISME J. 12, 2655-2667 (2018).

59. Beinart, R. A., Rotterová, J., Čepička, I., Gast, R. J. \& Edgcomb, V. P. The genome of an endosymbiotic methanogen is very similar to those of its free-living relatives. Environ. Microbiol. 20, 2538-2551 (2018).

60. Gutiérrez, G. Draft genome sequence of Methanobacterium formicicum DSM 3637, an archaebacterium isolated from the methane producer amoeba Pelomyxa palustris. (2012).

61. Berg, G., Grube, M., Schloter, M. \& Smalla, K. Unraveling the plant microbiome: looking back and future perspectives. Front. Microbiol. 5, 148 (2014).

62. Taffner, J., Cernava, T., Erlacher, A. \& Berg, G. Novel insights into plant-associated archaea and their functioning in arugula (Eruca sativa Mill.). J. Adv. Res. (2019).

63. Lu, Y. \& Conrad, R. In situ stable isotope probing of methanogenic archaea in the rice rhizosphere. Science (80-. ). 309, 1088-1090 (2005).

64. Lee, H. J., Jeong, S. E., Kim, P. J., Madsen, E. L. \& Jeon, C. O. High resolution depth distribution of Bacteria, Archaea, methanotrophs, and methanogens in the bulk and rhizosphere soils of a flooded rice paddy. Front. Microbiol. 6, 639 (2015).

65. Pump, J., Pratscher, J. \& Conrad, R. Colonization of rice roots with methanogenic archaea controls photosynthesis-derived methane emission. Environ. Microbiol. 17, 2254-2260 (2015). 
66. Joabsson, A., Christensen, T. R. \& Wallén, B. Vascular plant controls on methane emissions from northern peatforming wetlands. Trends Ecol. Evol. 14, 385-388 (1999).

67. Conrad, R. The global methane cycle: recent advances in understanding the microbial processes involved. Environ. Microbiol. Rep. 1, 285-292 (2009).

68. Müller, H. et al. Plant genotype-specific archaeal and bacterial endophytes but similar Bacillus antagonists colonize Mediterranean olive trees. Front. Microbiol. 6, (2015).

69. Taffner, J., Bergna, A., Cernava, T. \& Berg, G. Tomato-associated archaea show a cultivar-specific rhizosphere effect but an unspecific transmission by seeds. Phytobiomes J. (2020).

70. Song, G. C. et al. Plant growth-promoting archaea trigger induced systemic resistance in Arabidopsis thaliana against Pectobacterium carotovorum and Pseudomonas syringae. Environ. Microbiol. 21, 940948 (2019).

71. Hallam, S. J. et al. Genomic analysis of the uncultivated marine crenarchaeote Cenarchaeum symbiosum. Proc. Natl. Acad. Sci. 103, 18296-18301 (2006).

72. Preston, C. M., Wu, K. Y., Molinski, T. F. \& DeLong, E. F. A psychrophilic crenarchaeon inhabits a marine sponge: Cenarchaeum symbiosum gen. nov., sp. nov. Proc. Natl. Acad. Sci. 93, 6241-6246 (1996).

73. Jackson, S. A. et al. Archaea appear to dominate the microbiome of Inflatella pellicula deep sea sponges. PLoS One 8, e84438 (2013).

74. Kinsman, R., Sauer, F. D., Jackson, H. A. \& Wolynetz, M. S. Methane and carbon dioxide emissions from dairy cows in full lactation monitored over a six-month period. J. Dairy Sci. 78, 2760-2766 (1995).

75. Duin, E. C. et al. Mode of action uncovered for the specific reduction of methane emissions from ruminants by the small molecule 3-nitrooxypropanol. Proc. Natl. Acad. Sci. U. S. A. 113, 6172-6177 (2016).

76. Shabat, S. K. Ben et al. Specific microbiome-dependent mechanisms underlie the energy harvest efficiency of ruminants. ISME J. 10, 2958-2972 (2016).

77. Nauer, P. A., Hutley, L. B. \& Arndt, S. K. Termite mounds mitigate half of termite methane emissions. Proc. Natl. Acad. Sci. U. S. A. 115, 13306-13311 (2018).

78. Oxley, A. et al. Halophilic archaea in the human intestinal mucosa. Environ. Microbiol. 12, 2398-2410 (2010).

79. Liu, X. et al. Insights into the ecology, evolution, and metabolism of the widespread Woesearchaeotal lineages. Microbiome 6, 102 (2018).

80. Arumugam, M. et al. Enterotypes of the human gut microbiome. Nature 473, 174-180 (2013).

81. Hajishengallis, G., Darveau, R. P. \& Curtis, M. A. The keystone-pathogen hypothesis. Nat. Rev. Microbiol. 10, 717-725 (2012).

82. Dridi, B., Henry, M., El Khechine, A., Raoult, D. \& Drancourt, M. High prevalence of Methanobrevibacter smithii and Methanosphaera stadtmanae detected in the human gut using an improved DNA detection protocol. PLoS One 4, e7063-e7063 (2009).

83. Polag, D. \& Keppler, F. Global methane emissions from the human body: Past, present and future. Atmos. Environ. 214, 116823 (2019).

Summarizes the current knowledge on the methane emission of children and adults in different countries and calculates the level of human methane production over time.

84. Saunois, M. et al. The global methane budget 2000-2012. Earth Syst. Sci. Data 8, 697-751 (2016).

85. Gottlieb, K. et al. Selection of a cut-off for high-and low-methane producers using a spot-methane breath test: results from a large north American dataset of hydrogen, methane and carbon dioxide measurements in breath. Gastroenterol. Rep. gow048 (2017).

86. Morii, H., Oda, K., Suenaga, Y. \& Nakamura, T. Low methane concentration in the breath of Japanese. J. 
UOEH 25, 397-407 (2003).

87. Segal, I., Walker, A. R., Lord, S. \& Cummings, J. H. Breath methane and large bowel cancer risk in contrasting African populations. Gut 29, 608-613 (1988).

88. Hudson, M. J., Tomkins, A. M., Wiggins, H. S. \& Drasar, B. S. Breath methane excretion and intestinal methanogenesis in children and adults in rural Nigeria. Scand. J. Gastroenterol. 28, 993-998 (1993).

89. O'Keefe, S. J. D. et al. Why do African Americans get more colon cancer than Native Africans? J. Nutr. 137, 175S-182S (2007).

90. Nava, G. M. et al. Hydrogenotrophic microbiota distinguish native Africans from African and European Americans. Environ. Microbiol. Rep. 4, 307-315 (2012).

91. Levitt, M. D., Furne, J. K., Kuskowski, M. \& Ruddy, J. Stability of human methanogenic flora over 35 years and a review of insights obtained from breath methane measurements. Clin. Gastroenterol. Hepatol. 4, 123-129 (2006).

92. Weaver, G. A., Krause, J. A., Miller, T. L. \& Wolin, M. J. Incidence of methanogenic bacteria in a sigmoidoscopy population: an association of methanogenic bacteria and diverticulosis. Gut 27, 698-704 (1986).

93. Stewart, J. A., Chadwick, V. S. \& Murray, A. Carriage, quantification, and predominance of methanogens and sulfate-reducing bacteria in faecal samples. Lett. Appl. Microbiol. 43, 58-63 (2006).

94. Mihajlovski, A., Doré, J., Levenez, F., Alric, M. \& Brugère, J. Molecular evaluation of the human gut methanogenic archaeal microbiota reveals an age-associated increase of the diversity. Environ. Microbiol. Rep. 2, 272-280 (2010).

95. Goodrich, J. K. et al. Genetic determinants of the gut microbiome in UK twins. Cell Host Microbe 19, 731-743 (2016).

96. Hansen, E. E. et al. Pan-genome of the dominant human gut-associated archaeon, Methanobrevibacter smithii, studied in twins. Proc. Natl. Acad. Sci. 108, 4599-4606 (2011).

97. Goodrich, J. K., Davenport, E. R., Clark, A. G. \& Ley, R. E. The Relationship Between the Human Genome and Microbiome Comes into View. Annu. Rev. Genet. 51, 413-433 (2017).

98. Bonder, M. J. et al. The effect of host genetics on the gut microbiome. Nat. Genet. 48, 1407-1412 (2016).

99. Goodrich, J. J. K. et al. Human genetics shape the gut microbiome. Cell 159, 789-799 (2014).

100. Ruaud, A. et al. Syntrophy via Interspecies H2 Transfer between Christensenella and Methanobrevibacter Underlies Their Global Cooccurrence in the Human Gut. MBio 11, (2020).

101. Hoffmann, C. et al. Archaea and fungi of the human gut microbiome: correlations with diet and bacterial residents. PLoS One 8, (2013).

102. Nam, Y.-D. et al. Bacterial, archaeal, and eukaryal diversity in the intestines of Korean people. J. Microbiol. 46, 491-501 (2008).

103. Khelaifia, S. \& Raoult, D. Haloferax massiliensis sp. nov., the first human-associated halophilic archaea. New Microbes New Infect. 12, 96-98 (2016).

104. Nkamga, V. D., Henrissat, B. \& Drancourt, M. Archaea: essential inhabitants of the human digestive microbiota. Hum. Microbiome J. 3, 1-8 (2017).

105. Samuel, B. S. et al. Genomic and metabolic adaptations of Methanobrevibacter smithii to the human gut. Proc. Natl. Acad. Sci. 104, 10643-10648 (2007).

Describes the level of adaptation of a human-associated archaeon to the human gastrointestinal tract.

106. Ng, F. et al. An adhesin from hydrogen-utilizing rumen methanogen Methanobrevibacter ruminantium M1 binds a broad range of hydrogen-producing microorganisms. Environ. Microbiol. 18(9), 3010-3021 (2016). 
107. Lurie-Weinberger, M. N., Peeri, M., Tuller, T. \& Gophna, U. Extensive inter-domain lateral gene transfer in the evolution of the human commensal Methanosphaera stadtmanae. Front. Genet. 3, 182 (2012).

108. Lurie-Weinberger, M. N., Peeri, M. \& Gophna, U. Contribution of lateral gene transfer to the gene repertoire of a gut-adapted methanogen. Genomics 99, 52-58 (2012).

109. Bang, C. et al. Biofilm formation of mucosa-associated methanoarchaeal strains. Front. Microbiol. 5, 353 (2014).

110. Lepp, P. W. et al. Methanogenic Archaea and human periodontal disease. Proc. Natl. Acad. Sci. U. S. A. 101, 6176-6181 (2004).

First in-depth analysis of the link between archaea and periodontitis and proposition of their involvement in this disease.

111. Nkamga, V. D. et al. Methanobrevibacter oralis detected along with Aggregatibacter actinomycetemcomitans in a series of community-acquired brain abscesses. Clin. Microbiol. Infect. Off. Publ. Eur. Soc. Clin. Microbiol. Infect. Dis. 24, 207 (2018).

112. Borrel, G. et al. Comparative genomics highlights the unique biology of Methanomassiliicoccales, a Thermoplasmatales-related seventh order of methanogenic archaea that encodes pyrrolysine. BMC Genomics 15, 679 (2014).

113. Jones, B. V, Begley, M., Hill, C., Gahan, C. G. M. \& Marchesi, J. R. Functional and comparative metagenomic analysis of bile salt hydrolase activity in the human gut microbiome. Proc. Natl. Acad. Sci. 105, 13580-13585 (2008).

114. Bang, C. et al. Effects of antimicrobial peptides on methanogenic archaea. Antimicrob. Agents Chemother. 56, 4123-4130 (2012).

115. Blais Lecours, P. et al. Immunogenic properties of archaeal species found in bioaerosols. PLoS One 6, e23326 (2011).

116. Vierbuchen, T., Bang, C., Rosigkeit, H., Schmitz, R. A. \& Heine, H. The human-associated archaeon Methanosphaera stadtmanae is recognized through its rna and induces Tlr8-Dependent nIrP3 inflammasome activation. Front. Immunol. 8, 1535 (2017).

Report on methanoarchaeal RNA as immune stimulator and identification of the methanoarchaeal receptor.

117. Haines, A., Dilawari, J., Metz, G., Blendis, L. \& Wiggins, H. Breath-methane in patients with cancer of the large bowel. Lancet 310, 481-483 (1977).

118. Mahnert, A., Blohs, M., Pausan, M. R. \& Moissl-Eichinger, C. The human archaeome: methodological pitfalls and knowledge gaps. Emerg. Top. Life Sci. 2.4, 469-482 (2018).

119. Belay, N., Mukhopadhyay, B., Conway de Macario, E., Galask, R. \& Daniels, L. Methanogenic bacteria in human vaginal samples. J. Clin. Microbiol. 28, 1666-1668 (1990).

120. Grine, G. et al. Detection of Methanobrevibacter smithii in vaginal samples collected from women diagnosed with bacterial vaginosis. Eur. J. Clin. Microbiol. Infect. Dis. 1-7 (2019).

121. Pérez-Cobas, A. E., Ginevra, C., Rusniok, C., Jarraud, S. \& Buchrieser, C. Legionella pneumophila infection and antibiotic treatment engenders a highly disturbed pulmonary microbiome with decreased microbial diversity. bioRxiv 808238 (2019).

122. Grine, G. et al. Co-culture of Methanobrevibacter smithii with enterobacteria during urinary infection. EBioMedicine 43, 333-337 (2019).

123. Sogodogo, E. et al. Nine Cases of Methanogenic Archaea in Refractory Sinusitis, an Emerging Clinical Entity. Front. public Heal. 7, (2019).

124. Drancourt, M. et al. Evidence of Archaeal Methanogens in Brain Abscess. Clin. Infect. Dis. 65, 1-5 (2017). 
125. Nguyen-Hieu, T., Khelaifia, S., Aboudharam, G. \& Drancourt, M. Methanogenic archaea in subgingival sites: a review. APMIS 121, 467-77 (2013).

126. Belkacemi, S. et al. Peri-implantitis-associated methanogens: a preliminary report. Sci. Rep. 8, 9447 (2018).

127. Vianna, M. E., Holtgraewe, S., Seyfarth, I., Conrads, G. \& Horz, H. P. Quantitative analysis of three hydrogenotrophic microbial groups, methanogenic archaea, sulfate-reducing bacteria, and acetogenic bacteria, within plaque biofilms associated with human periodontal disease. J. Bacteriol. 190, 37793785 (2008).

128. Siqueira, J. F. J. \& Rocas, I. N. Community as the unit of pathogenicity: an emerging concept as to the microbial pathogenesis of apical periodontitis. Oral Surg. Oral Med. Oral Pathol. Oral Radiol. Endod. 107, 870-878 (2009).

129. de Macario, E. C. \& Macario, A. J. L. Methanogenic archaea in health and disease: a novel paradigm of microbial pathogenesis. Int. J. Med. Microbiol. 299, 99-108 (2009).

130. Benoit, S. L., Maier, R. J., Sawers, R. G. \& Greening, C. Molecular Hydrogen Metabolism: a Widespread Trait of Pathogenic Bacteria and Protists. Microbiol. Mol. Biol. Rev. 84, (2020).

131. Lecours, P. B. et al. Increased prevalence of methanosphaera stadtmanae in inflammatory bowel diseases. PLoS One 9, 1-7 (2014).

132. Barnett, D. J. M., Mommers, M., Penders, J., Arts, I. C. W. \& Thijs, C. Intestinal archaea inversely associated with childhood asthma. J. Allergy Clin. Immunol. 143, 2305-2307 (2019).

133. Pimentel, M. Methane, a gas produced by enteric bacteria, slows intestinal transit and augments small intestinal contractile activity. AJP Gastrointest. Liver Physiol. 290, G1089-G1095 (2006).

134. Chatterjee, S., Park, S., Low, K., Kong, Y. \& Pimentel, M. The degree of breath methane production in IBS correlates with the severity of constipation. Am. J. Gastroenterol. 102, 837 (2007).

135. Attaluri, A., Jackson, M., Valestin, J. \& Rao, S. S. C. Methanogenic flora is associated with altered colonic transit but not stool characteristics in constipation without IBS. Am. J. Gastroenterol. 105, 1407 (2010).

136. Tottey, W. et al. Colonic transit time is a driven force of the gut microbiota composition and metabolism: in vitro evidence. J. Neurogastroenterol. Motil. 23, 124 (2017).

137. Lurie-Weinberger, M. N. \& Gophna, U. Archaea in and on the human body: health implications and future directions. PLOS Pathog. 11, e1004833 (2015).

138. Gottlieb, K., Wacher, V., Sliman, J. \& Pimentel, M. inhibition of methanogenic archaea by statins as a targeted management strategy for constipation and related disorders. Aliment. Pharmacol. Ther. 43, 197-212 (2016).

139. Jia, Y., Li, Z., Liu, C. \& Zhang, J. Methane Medicine: A Rising Star Gas with Powerful Anti-Inflammation, Antioxidant, and Antiapoptosis Properties. Oxid. Med. Cell. Longev. 2018, 1912746 (2018).

140. Xin, L., Sun, X. \& Lou, S. Effects of Methane-Rich Saline on the Capability of One-Time Exhaustive Exercise in Male SD Rats. PLoS One 11, e0150925 (2016).

141. Laverdure, R., Mezouari, A., Carson, M. A., Basiliko, N. \& Gagnon, J. A role for methanogens and methane in the regulation of GLP-1. Endocrinol. diabetes Metab. 1, e00006 (2018).

142. Boros, M. \& Keppler, F. Methane Production and Bioactivity-A Link to Oxido-Reductive Stress. Front. Physiol. 10, 1244 (2019).

143. Al-Waiz, M., Mikov, M., Mitchell, S. C. \& Smith, R. L. The exogenous origin of trimethylamine in the mouse. Metabolism 41, 135-136 (1992).

144. Wang, Z. et al. Gut flora metabolism of phosphatidylcholine promotes cardiovascular disease. Nature 472, 57 (2011).

145. Tang, W. H. W. et al. Gut microbiota-dependent trimethylamine N-oxide (TMAO) pathway contributes 
to both development of renal insufficiency and mortality risk in chronic kidney disease. Circ. Res. 116, 448-455 (2015).

146. Mackay, R. J., McEntyre, C. J., Henderson, C., Lever, M. \& George, P. M. Trimethylaminuria: causes and diagnosis of a socially distressing condition. Clin. Biochem. Rev. 32, 33 (2011).

147. Srinivasan, G., James, C. M. \& Krzycki, J. A. Pyrrolysine encoded by UAG in Archaea: charging of a UAGdecoding specialized tRNA. Science (80-. ). 296, 1459-1462 (2002).

148. Gaston, M. A., Jiang, R. \& Krzycki, J. A. Functional context, biosynthesis, and genetic encoding of pyrrolysine. Curr. Opin. Microbiol. 14, 342-349 (2011).

149. Brugère, J.-F., Atkins, J. F., O’Toole, P. W. \& Borrel, G. Pyrrolysine in archaea: a 22nd amino acid encoded through a genetic code expansion. Emerg. Top. Life Sci. 2, 607-618 (2018).

150. Dridi, B., Fardeau, M.-L. M.-L., Ollivier, B., Raoult, D. \& Drancourt, M. Methanomassiliicoccus luminyensis gen. nov., sp. nov., a methanogenic archaeon isolated from human faeces. Int. J. Syst. Evol. Microbiol. 62, 1902-1907 (2012).

151. Ramezani, A. et al. Gut Colonization with Methanogenic Archaea Lowers Plasma Trimethylamine Noxide Concentrations in Apolipoprotein e-/- Mice. Sci. Rep. 8, 14752 (2018).

152. Fadhlaoui, K. et al. Archaea, specific genetic traits, and development of improved bacterial live biotherapeutic products: another face of next-generation probiotics. Appl. Microbiol. Biotechnol. 104, 4705-4716 (2020).

153. Prangishvili, D. et al. Sulfolobicins, specific proteinaceous toxins produced by strains of the extremely thermophilic archaeal genus Sulfolobus. J. Bacteriol. 182, 2985-2988 (2000).

154. Martin, W. Pathogenic archaebacteria: do they not exist because archaebacteria use different vitamins? BioEssays 26, 592-593 (2004).

155. Cavicchioli, R. \& Curmi, P. Response to William Martin's letter. (2004).

156. Gill, E. E. \& Brinkman, F. S. L. The proportional lack of archaeal pathogens: Do viruses/phages hold the key? BioEssays 33, 248-254 (2011).

157. Prangishvili, D. et al. The enigmatic archaeal virosphere. Nat. Rev. Microbiol. 15, 724-739 (2017).

158. Microbiology by numbers. Nat. Rev. Microbiol. 9, 628 (2011).

159. Sachs, J. L., Skophammer, R. G. \& Regus, J. U. Evolutionary transitions in bacterial symbiosis. Proc. Natl. Acad. Sci. U. S. A. 108 Suppl, 10800-10807 (2011).

160. Valentine, D. L. Adaptations to energy stress dictate the ecology and evolution of the Archaea. Nat. Rev. Microbiol. 5, 316-23 (2007).

161. Castelle, C. J. \& Banfield, J. F. Major New Microbial Groups Expand Diversity and Alter our Understanding of the Tree of Life. Cell 172, 1181-1197 (2018).

162. Borrel, G. et al. Wide diversity of methane and short-chain alkane metabolisms in uncultured archaea. Nat. Microbiol. 4, 603-613 (2019).

163. Anonymous. Statistical Yearbook of the Food and Agriculture Organisation for the United Nations. (2016).

164. Monteiro, A. L. G. et al. The role of small ruminants on global climate change. Acta Sci. Anim. Sci. 40, (2018).

165. Sanderson, M. G. Biomass of termites and their emissions of methane and carbon dioxide: A global database. Global Biogeochem. Cycles 10, 543-557 (1996).

166. Daemmgen, U. et al. Enteric methane emissions from German pigs. Agric. For. Res. 3, 83-96 (2012).

167. Brouček, J. \& Čermák, B. Emission of harmful gases from poultry farms and possibilities of their reduction. Ekologia 34, 89-100 (2015).

168. Almeida, A. et al. A new genomic blueprint of the human gut microbiota. Nature 568, 499 (2019). 
169. Quast, C. et al. The SILVA ribosomal RNA gene database project: improved data processing and webbased tools. Nucleic Acids Res. 41, D590-D596 (2013).

170. Albers, S.-V. \& Meyer, B. H. The archaeal cell envelope. Nat. Rev. Microbiol. 9, 414 (2011).

171. Becker, K. W. et al. Unusual butane-and pentanetriol-based tetraether lipids in Methanomassiliicoccus luminyensis, a representative of the seventh order of methanogens. Appl. Environ. Microbiol. 82, 45054516 (2016).

172. Klingl, A. S-layer and cytoplasmic membrane - exceptions from the typical archaeal cell wall with a focus on double membranes. Front. Microbiol. 5, 1-6 (2014).

173. Shahapure, R., Driessen, R. P. C., Haurat, M. F., Albers, S. \& Dame, R. T. The archaellum: a rotating type IV pilus. Mol. Microbiol. 91, 716-723 (2014).

174. Chaudhury, P., Quax, T. E. F. \& Albers, S. Versatile cell surface structures of archaea. Mol. Microbiol. 107, 298-311 (2018).

175. Quemin, E. R. J. et al. First insights into the entry process of hyperthermophilic archaeal viruses. J. Virol. 87, 13379-13385 (2013).

176. Sato, T. \& Atomi, H. Novel metabolic pathways in Archaea. Curr. Opin. Microbiol. 14, 307-314 (2011).

177. Brasen, C., Esser, D., Rauch, B. \& Siebers, B. Carbohydrate metabolism in Archaea: current insights into unusual enzymes and pathways and their regulation. Microbiol. Mol. Biol. Rev. 78, 89-175 (2014).

178. Leininger, S. et al. Archaea predominate among ammonia-oxidizing prokaryotes in soils. Nature 442, 806-809 (2006).

179. Stein, L. Y. Insights into the physiology of ammonia-oxidizing microorganisms. Curr. Opin. Chem. Biol. 49, 9-15 (2019).

180. Mand, T. D. \& Metcalf, W. W. Energy Conservation and Hydrogenase Function in Methanogenic Archaea, in Particular the Genus Methanosarcina. Microbiol. Mol. Biol. Rev. 83, (2019).

181. Borrel, G., Adam, P. S. \& Gribaldo, S. Methanogenesis and the Wood-Ljungdahl pathway: an ancient, versatile, and fragile association. Genome Biol. Evol. evw114 (2016).

182. Stams, A. J. M. \& Plugge, C. M. Electron transfer in syntrophic communities of anaerobic bacteria and archaea. Nat. Rev. Microbiol. 7, 568-577 (2009).

183. Ettwig, K. F. et al. Archaea catalyze iron-dependent anaerobic oxidation of methane. Proc. Natl. Acad. Sci. U. S. A. 113, 12792-12796 (2016).

184. Cai, C. et al. A methanotrophic archaeon couples anaerobic oxidation of methane to $\mathrm{Fe}(\mathrm{III})$ reduction. ISME J. 12, 1929-1939 (2018).

185. Scheller, S., Yu, H., Chadwick, G. L., McGlynn, S. E. \& Orphan, V. J. Artificial electron acceptors decouple archaeal methane oxidation from sulfate reduction. Science 351, 703-707 (2016).

186. Wegener, G., Krukenberg, V., Riedel, D., Tegetmeyer, H. E. \& Boetius, A. Intercellular wiring enables electron transfer between methanotrophic archaea and bacteria. Nature 526, 587-590 (2015).

187. Kiener, A., Konig, H., Winter, J. \& Leisinger, T. Purification and use of Methanobacterium wolfei pseudomurein endopeptidase for lysis of Methanobacterium thermoautotrophicum. J. Bacteriol. 169, 1010-1016 (1987).

188. Lee, Z. \& Bussema, C. Schmidt, T. rrnDB: documenting the number of rRNA and tRNA genes in bacteria and archaea. Nucleic Acids Res 37, D489-D493 (2009).

189. Sun, Y., Liu, Y., Pan, J., Wang, F. \& Li, M. Perspectives on Cultivation Strategies of Archaea. Microb. Ecol. (2019). doi:10.1007/s00248-019-01422-7 


\section{Acknowledgements}

The authors gratefully acknowledge A. Mahnert for support in the preparation of Figure 3, and M. Blohs for input on methane production in humans. Funding given by the Austrian Science Fund (FWF) to C.M.-E. (Project IDs P 30796 and P 32697) is highly appreciated, as is the funding from the German Science Foundation (DFG) given to R.A.S. (SCHM1052/11-1/2). The French National Agency for Research is greatly acknowledged for funding to G.B. and S.G. (Grants ArchEvol ANR-16-CE02-0005-01 and Methevol ANR-19-CE02-0005-01) and J.F.B. ('Investissements d'Avenir' 16-IDEX-0001 CAP 20-25, grant from Hub Innovergne).

\section{Author contributions}

The authors contributed equally to all aspects of the article.

\section{Competing interests}

The authors declare no competing interests.

\section{Peer review information}

Nature Reviews Microbiology thanks U. Gophna, I. Mizrahi, D. Relman and the other, anonymous, reviewer(s) for their contribution to the peer review of this work.

\section{Publisher's note}

Springer Nature remains neutral with regard to jurisdictional claims in published maps and institutional affiliations.

\section{Supplementary information}

Supplementary information is available for this paper at https://doi.org/10.1038/s415XX-XXX-XXXX-X 


\section{Related links}

The Food and Agriculture Organization: www.FAO.org

Fig. 1: Archaeal diversity. Shown is a schematic of the current tree of Archaea based on the most recent phylogenomic analyses ${ }^{12,13,161,162}$, and host-associated clades, including Methanosarcinales, Methanomassiliicocci, Thaumarchaeota, Halobacteria, Methanocellales, Methanomicrobiales, Methanobacteria and Woesearchaeota, are highlighted. Host-associated archaea are phylogenetically diverse, but are mostly composed of methanogens and, more recently, Thaumarchaeota. The most basal phylogenetic relationships, including the monophyly of Euryarchaeota and DPANN, are still under debate ${ }^{12}$.

Fig. 2: Methane emission in cattle in comparison to other animals. Methane $\left(\mathrm{CH}_{4}\right)$ is formed by the methanoarchaeal components of the rumen and protist microbiome. The archaea syntrophically consume hydrogen $\left(\mathrm{H}_{2}\right)$, a product of anaerobic fermentation, and hereby support the metabolic activity of bacteria and protists. The methanogens can be free-living in the rumen, but specifically members of the orders Methanobacteriales, Methanomicrobiales, Methanosarcinales and possibly Methanomassiliicoccales can live as endosymbionts in the cytoplasm of anaerobic protists. These endosymbiotic methanogens utilize the $\mathrm{H}_{2}$ that is generated by hydrogenosomes following the oxidation of pyruvate for methanogenesis. Methane is mainly emitted by eructation of cattle and by flatulence. The shows a comparison of the global methane emission rates of cattle $^{163,164}$, termites ${ }^{165}$, sheep ${ }^{163,164}$, goats ${ }^{163,164}$, pigs ${ }^{166}$ (considering one billion pigs worldwide (The Food and Agriculture Organization [www.FAO.org]), humans ${ }^{83}$, chicken ${ }^{167}$ (considering 23 billion chicken worldwide; The Food and Agriculture Organization [www.FAO.org]) in million tons per year (mtpa). Methane levels from cattle released into the atmosphere are the highest amongst the livestock, and represent one of the largest sources of anthropogenic methane emissions.

Fig. 3: Archaeal taxa detected in human, animal and plant samples. $16 \mathrm{~S}$ rRNA gene sequences from isolated strains, publicly available clone sequences (for example, Ref. ${ }^{16}$ ), reconstructed metagenome assembled genomes (MAGs) from human microbiomes ${ }^{15,168}$ and sequences from amplicon-based studies of animals ${ }^{23,24}$, humans ${ }^{17,20}$ and plants ${ }^{22,62,68,69}$ were quality filtered (no singletons, length $>100 \mathrm{bp}$, alignment score $>30$, alignment identity $>40 \%$; ${ }^{169}$ ), grouped at $97 \%$ similarity, and processed through SILVA SINA classification. Trees were calculated via RAxML, on the backbone of three neighbour sequences per query which were used to stabilize the tree ('add to neighbours tree' option; neighbor representatives are shown in the tree with an unlabeled node) (for a detailed overview please see supplementary figures). For the human archaeome tree (top panel), lineages found in only one publication are not shown; this filtering was not applied for the animal archaeome tree (bottom left panel) and plant archaeome tree (bottom right panel) due to the small number of available studies. Output was completed with meta-information (sample origin, isolate) using Itol ${ }^{169}$. Thaumarchaeota (correspond to Nitrososphaeria, in shades of orange), Woesearchaeota (in very soft red), and Halobacteriales ( in shades of grey ), Methanomicrobiales (in shades of red), Methanocellales (in shades of dark blue), Methanosarcinales (in shades of blue), Methanomassiliicoccales (in shades of purple), and Methanobacteriales (in shades of green), were found in all groups in different sample types (that is, skin, gastrointestinal tract (GIT) (including faeces, gut biopsies and rumen samples), respiratory tract and oral cavity samples), as well as green plant and/or seed samples, as indicated by the circles outside, which is linked to an individual archaeal representative. 
Fig. 4: Detailed information on five archaeal genera found in humans, animals and plants. The reported association of Halococcus, Methanobrevibacter, Methanosphaera, Methanosarcina and Methanoculleus with specific animals and plants in specific sample types is displayed. The sub-class taxonomic information is given for groups of animals and plants. Figure is based on the data of Fig. 3. GIT, gastrointestinal tract.

Figure 5: Interaction of the gastrointestinal archaeome with the host and the bacterial microbial community. The host provides a stable biotope, including nutrition, to the archaeal community and regulates the composition of the microbial community through antimicrobial peptides (AMPs) and bile acids. In addition, the host has been shown to release pro-inflammatory cytokines in response to some archaeal components. Archaea in the human gut may exhibit a high degree of specialized adaptation, which are mirrored by a number of specific traits, such as reduced and/or adapted physiological capacity (not shown) and defense mechanisms (adhesin-like proteins (ALPs), glycans, bile salt hydrolases and biofilm formation. Methanogenic archaea produce methane, a potential neuro- and immunomodulator (see main text), which is excreted by the host, contributing to the global methane emission (see figure 2), and affecting human physiology, such as gut motility. The host-associated bacteriome provides substrates for the archaeome (including formate, trimethylamine (TMA), methanol, $\mathrm{H}_{2}$ and $\mathrm{CO}_{2}$ ). Moreover, the bacteriome may be a source of genetic material for archaeal members of the microbiome via horizontal gene transfer (HGT), allowing the acquisition of traits like ALPs, glycosyltransferases and bile salt hydrolases (BSHs). The interactions of the bacteriome with the host are not shown in this simplified schematic. 


\section{Text box 1: The unique biology of Archaea}

At first glance, archaea resemble bacteria, as they lack a nucleus and organelles, possess circular genomes, an operon-based gene arrangement, and ribosomes of 70S type (see the table). However, archaea are evolutionarily distantly related to bacteria, which is reflected in their divergent 16S rRNA sequences. Moreover, they share many traits with eukaryotes, particularly their molecular machineries for transmission and manipulation of genetic information (for example, RNA and DNA polymerases).

Archaea also possess specific structural characteristics, such as unique membrane lipids $\left(C_{5}\right.$ isoprenoid units ether-coupled to L-glycerol at the $(s n)-2,3$ position $)^{170,171}$. Their cell envelopes never contain bacterial-like peptidoglycan or lipopolysaccharides but can be composed of protein, pseudomurein or modified heteropolysaccharides. In some cases, even only single or double membranes without additional layers function as an outer shell ${ }^{172}$. Consequently, archaea are not affected by antibiotics that target peptidoglycan, such as $\beta$-lactams, but are susceptible to antimicrobials that are also active against both bacteria and eukaryotes, such as metronidazole ${ }^{34}$.

For motility, archaea use unique rotating flagella ('archaella') that are not homologous to those of bacteria and eukaryotes, but evolutionarily and structurally related to type IV pili ${ }^{173}$. Archaeal surfaces can additionally bear pili or fimbriae, cannulae, fibers or even grappling hook-bearing hami ${ }^{174}$. Many of these cell surface structures enable them to interact with surfaces, other (microbial) cells or viruses ${ }^{175}$. Also, most archaea have a single membrane, and lack the specific machineries that many Gram-negative bacteria use to deliver toxins into eukaryotic target cells, such as type III secretion systems ${ }^{170}$.

Archaea can survive challenging conditions by switching to extreme slow growth or dormancy, but capacity to form spores has not been observed to date. They are widespread in various ecosystems and include autotrophs, heterotrophs, phototrophs, chemotrophs, organotrophs and lithotrophs, aerobes and anaerobes. 
Archaea are considered to comply with chronic energy stress, and are thus well adapted to nutrient-limiting ecological niches ${ }^{160}$.

Some of the archaea-specific pathways correspond to functions that are also present in bacteria (sugar metabolism, $\mathrm{CO}_{2}$ fixation and biosynthetic pathways) but involve archaea-specific enzymes ${ }^{176,177}$. This is exemplified by ammonia-oxidation ${ }^{178}$, a capacity with high ecological impact in marine and soil environments, whose pathway is distinct from its bacterial counterpart owing to the lack of heme-based enzymes ${ }^{179}$. Methane metabolisms (methanogenesis and methanotrophy), are widely distributed among archaea ${ }^{162}$, and involve a specific enzymatic complex, the methyl-coenzyme $M$ reductase. Methanogenesis is a unique metabolic trait of archaea that results in methane production via four main pathways: two of them commonly use hydrogen as electron donor (hydrogenotrophic pathways), that is, $\mathrm{CO}_{2}$-reducing and methyl-reducing methanogenesis, whereas the other two do not require an external hydrogen source and include methylotrophic and acetoclastic methanogenesis ${ }^{180,181}$. Methanogens are strict anaerobes and are widely distributed in various environments, such as freshwater and marine sediments, (wetland) soils and the digestive tract of animals. Methanogenesis relies on a limited number of simple compounds (for example, $\mathrm{H}_{2}$, $\mathrm{CO}_{2}$, formate, methyl-compounds and acetate), that are metabolic by-products of organic matter degradation in anoxic environments. By keeping $\mathrm{H}_{2}$ concentrations low, methanogens enable secondary fermentation (for example the utilization of volatile fatty acids, lactate and alcohol) to remain thermodynamically favorable and they increase the energy yield of primary fermenters using complex molecules such as carbohydrates ${ }^{182}$. Thus, by contributing to the overall efficiency of energy retrieval during digestion of organic matter, methanogens are considered to represent key-stone microorganisms in anoxic ecosystems. The contribution of methanogenesis to climate change is observed with concern, as most of biologically produced, atmospheric methane originates from archaeal metabolism ( 69\%; Ref. ${ }^{67}$ ). Methanotrophic archaea play an important role in the mitigation of methane emissions to the atmosphere, particularly from marine sediments. In contrast to 
bacterial methanotrophy, the archaeal pathway relies on the reverse use of the enzymes involved in methanogenesis. Moreover, some archaea couple methane oxidation with the direct reduction of electron acceptors not used by bacteria, such as iron and manganese oxides, nitrate, humic acids ${ }^{183-185}$ or with indirect electron transfer to sulfur compounds via sulfate-reducing bacteria ${ }^{186}$.

\begin{tabular}{|c|c|c|c|}
\hline Feature & Bacteria & Archaea & Eukaryotes \\
\hline Nucleus & no & no & yes \\
\hline Organelles & no & no & yes \\
\hline Spliceosomal introns & no & no & yes \\
\hline Chromosome shape & $\begin{array}{l}\text { Circular and } \\
\text { linear }\end{array}$ & Circular & Linear \\
\hline Operons & yes & yes & Rare \\
\hline RNA polymerase & Bacteria-like & Eukaryote-like & $\begin{array}{l}\text { Eukaryote- } \\
\text { like }\end{array}$ \\
\hline DNA polymerase & Bacteria-like & Eukaryote-like & $\begin{array}{l}\text { Eukaryote- } \\
\text { like }\end{array}$ \\
\hline Ribosome type & $70 S$ & $70 \mathrm{~S}$ & $80 S$ \\
\hline $\begin{array}{l}\text { Translation start } \\
\text { (amino acid) }\end{array}$ & $\begin{array}{l}\text { Formylmethio } \\
\text { nine }\end{array}$ & Methionine & Methionine \\
\hline Histones & no & yes & yes \\
\hline Peptidoglycan & yes & $\stackrel{\text { no }}{\text { Pseudo PG in some }}$ & no \\
\hline Motility & $\begin{array}{l}\text { Bacteria-type } \\
\text { flagellum }\end{array}$ & $\begin{array}{l}\text { Archaea-type } \\
\text { flagellum } \\
\text { (archaellum) }\end{array}$ & $\begin{array}{l}\text { Eukarya-type } \\
\text { flagellum }\end{array}$ \\
\hline Lipopolysaccharide & yes & no & no \\
\hline Membrane lipids & $\begin{array}{l}\text { Ester-links } \\
\text { (glycerol-1- } \\
\text { phosphate } \\
\text { backbone) }\end{array}$ & $\begin{array}{l}\text { Ether-links } \\
\text { (glycerol-3- } \\
\text { phosphate } \\
\text { backbone) }\end{array}$ & $\begin{array}{l}\text { Ester-links } \\
\text { (glycerol-1- } \\
\text { phosphate } \\
\text { backbone) }\end{array}$ \\
\hline Methanogenesis & no & yes & no \\
\hline Oxygenic & yes & no & yes \\
\hline
\end{tabular}




\begin{tabular}{|l|c|c|c|}
\hline photosynthesis & & & \\
\hline Spores & yes & no & yes \\
\hline Human pathogenicity & yes & no & yes \\
\hline
\end{tabular}

For entries denoted as 'yes' the specific trait is present in either all or some members.

\section{Text box 2: Methodological challenges for studying the archaeome}

Due to the different physiological, structural and molecular properties of archaea, bacteria-centric methodologies applied to complex microbial communities often fail to detect the contribution of the archaeome. This concerns many aspects (see the figure), including visualization (for example, nucleic acidbased fluorescence in situ hybridization), cultivation and molecular quantitative analyses. For example, numerous commercial DNA extraction kits contain lysozyme, an enzyme that breaks bacterial peptidoglycan, but not archaeal pseudo-peptidoglycan. Moreover, due to the hardy nature of the (methano-)archaeal cell wall, in particular that of Methanobacteriales such as Methanobrevibacter, Methanosphaera and Methanobacterium, additional physical treatment, for example extended bead-beating, application of additional detergents or pseudo peptidoglycan endopeptidases ${ }^{187}$, is necessary for efficient cell lysis. Also, most of the so-called 'universal' $16 \mathrm{~S}$ rRNA primers fail to cover the broad archaeal diversity and are thus unable to detect certain archaeal lineages in specific sample types ${ }^{21,118}$, such as tissue samples. Moreover, $16 \mathrm{~S}$ rRNA gene copy per genome of bacteria (mean: 4.9) outnumbers that of archaea (mean: 1.7), resulting in lowering the estimation of real archaeal representation by $16 \mathrm{~S}$ rRNA gene profiling or quantitative PCR ${ }^{188}$. Disadvantageous bacteria:archaea ratios, particularly in the case of massive eukaryotic DNA background in the samples, also challenge primer-independent, shotgun-based metagenomic studies. This could be overcome by 
a physical enrichment of archaeal cells, or the depletion of host DNA using molecular capturing methods. Finally, due to the limited availability of well annotated genomes in underrepresented archaeal phyla, current databases often fail to correctly assign archaeal sequences ${ }^{118}$. Other issues concern the cultivability of nonextreme archaea in general ${ }^{189}$, and the still unclear medical relevance of archaea.

\section{Glossary:}

Holobiont: A multicellular Eukaryote together with its associated microbial communities.

Endosymbiotic: Endosymbiontic microorganisms live in the cells of another organism.

Ectosymbionts: Microorganisms living on the surface of another organism in a symbiotic relationship.

Pseudogenization: Conversion of a gene into a nonfunctional gene-like sequence in a symbiotic relationship.

Rhizosphere. Soil area around a plant root, influenced by root exudates and inhabited by a specific population of microorganisms.

Endosphere. Internal regions of plant tissues, which are inhabited by endophytic microorganisms.

Phyllosphere. All above-ground parts of plants, serving as habitat for microorganisms.

Heritable: Proportion of variance in the phenotype which can be attributed to genetic differences between individuals.

Biofilm: Microbial consortium attached to a surface or interface and organized in an extracellular matrix Horizontal gene transfer: A process by which genetic material is acquired from another organism (as opposed to vertical inheritance where genetic information is transmitted from parent to offspring).

Glycosyltransferases: Enzymes that catalyze the transfer of glycosyl (sugar) residues to an acceptor molecule Microorganism-associated molecular patterns: Conserved molecules characteristic for microbes, which are recognized by the immune system.

\section{Table of content:}

The archaeal community, the archaeome, is now growingly recognized as an important component of hostassociated microbiomes. In this Review, Moissl-Eichinger and colleagues discuss the diversity and potential role of archaea associated with protists, plants and animals, and they highlight the potential role of archaea in human health and disease. 


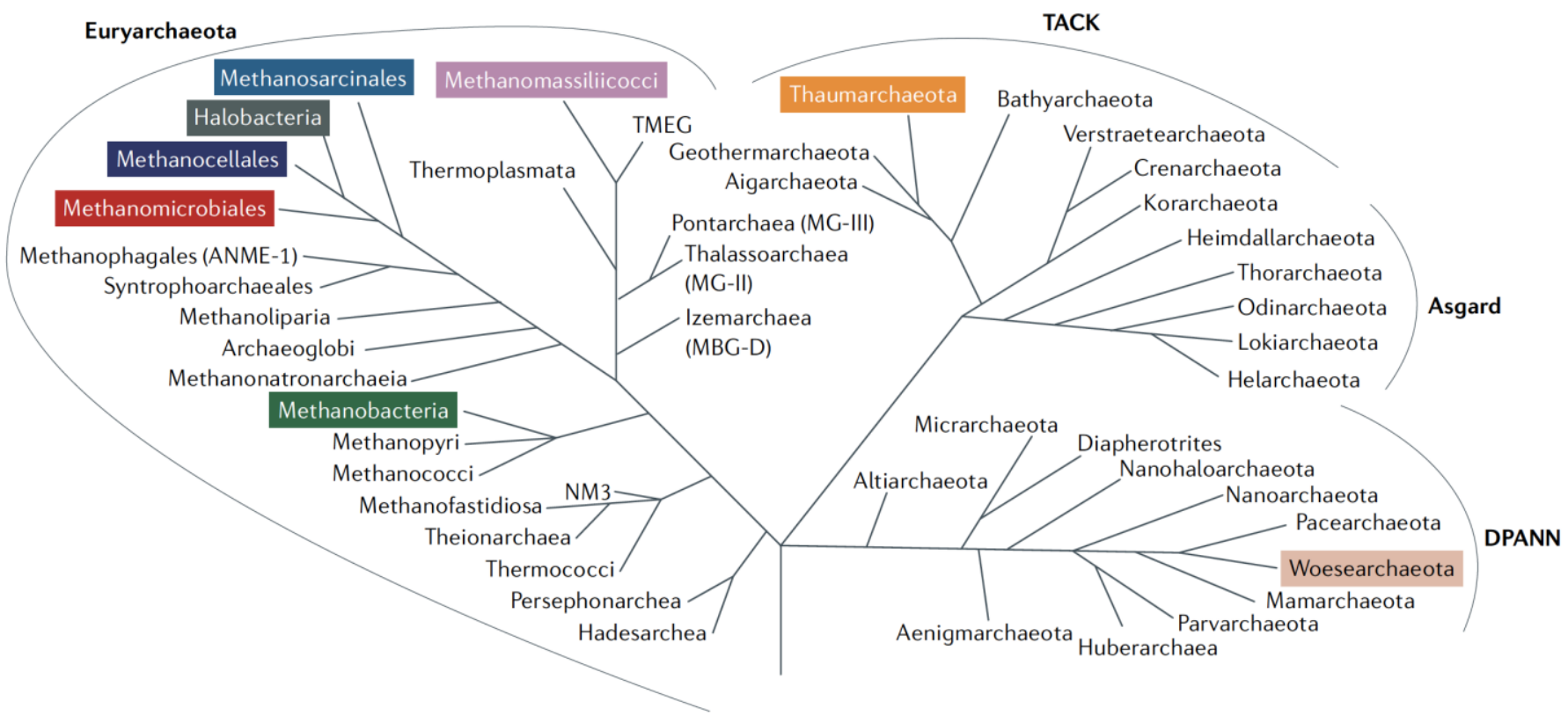

\section{Figure 1}




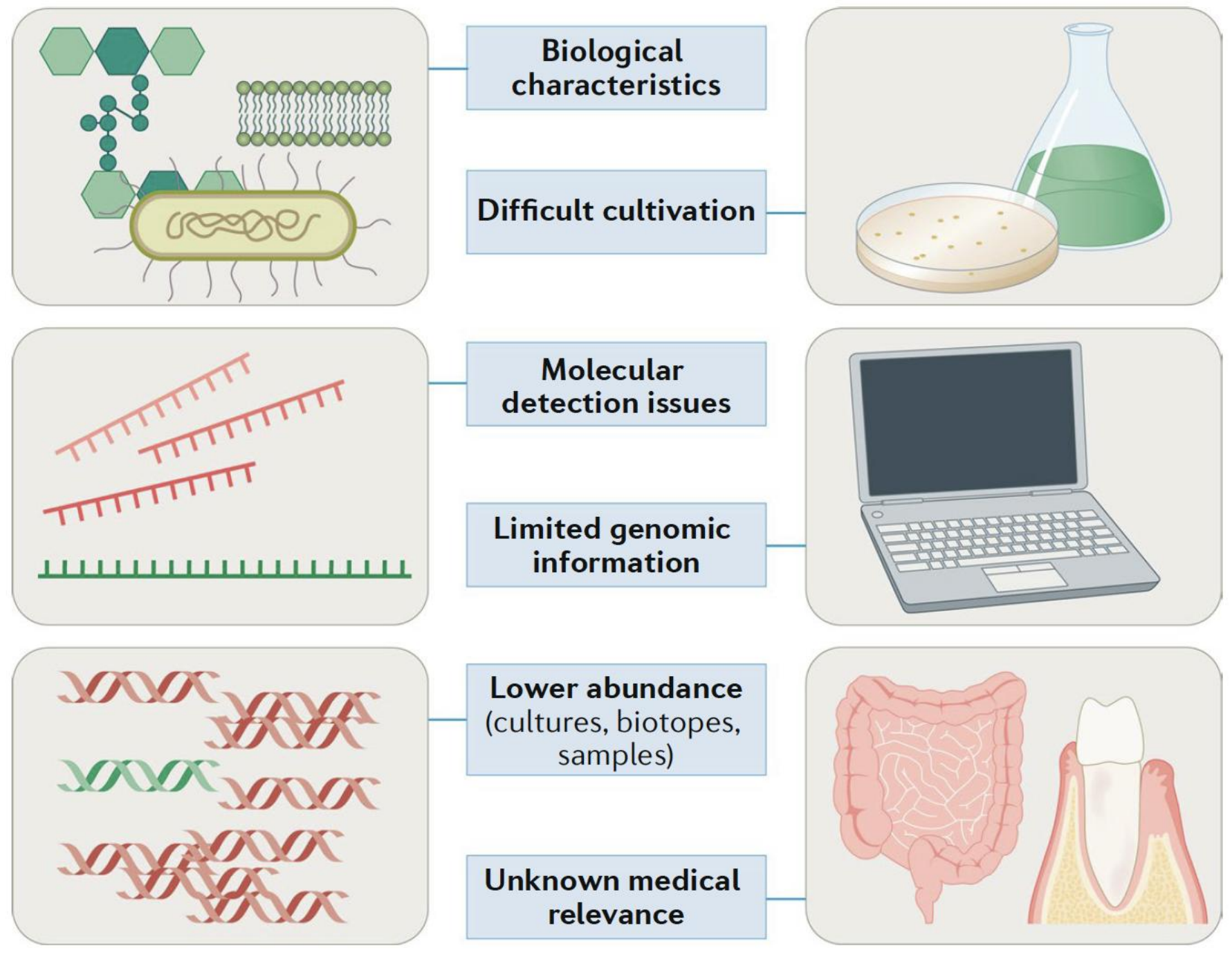

Figure Box 2 


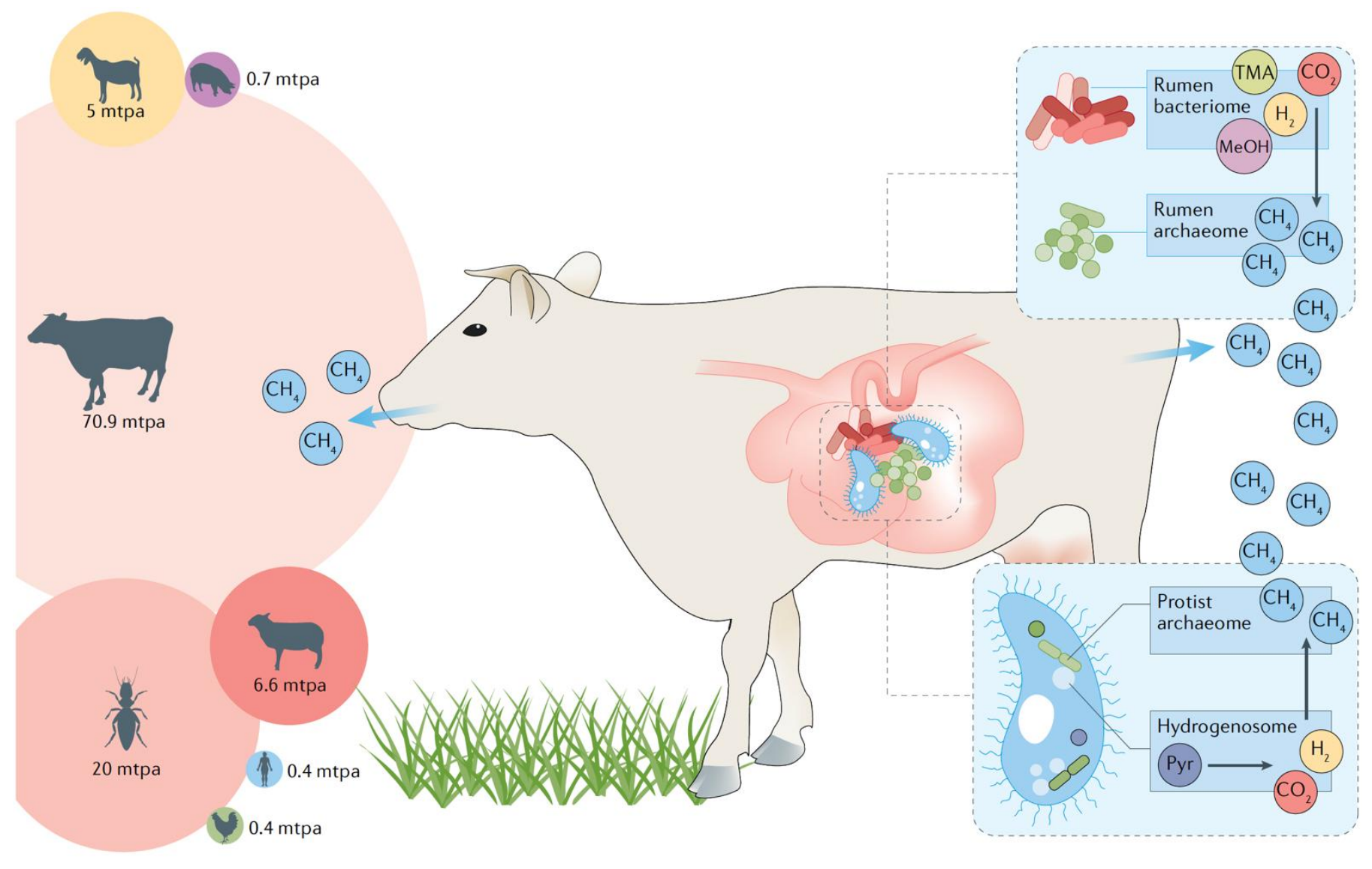

Figure 2 


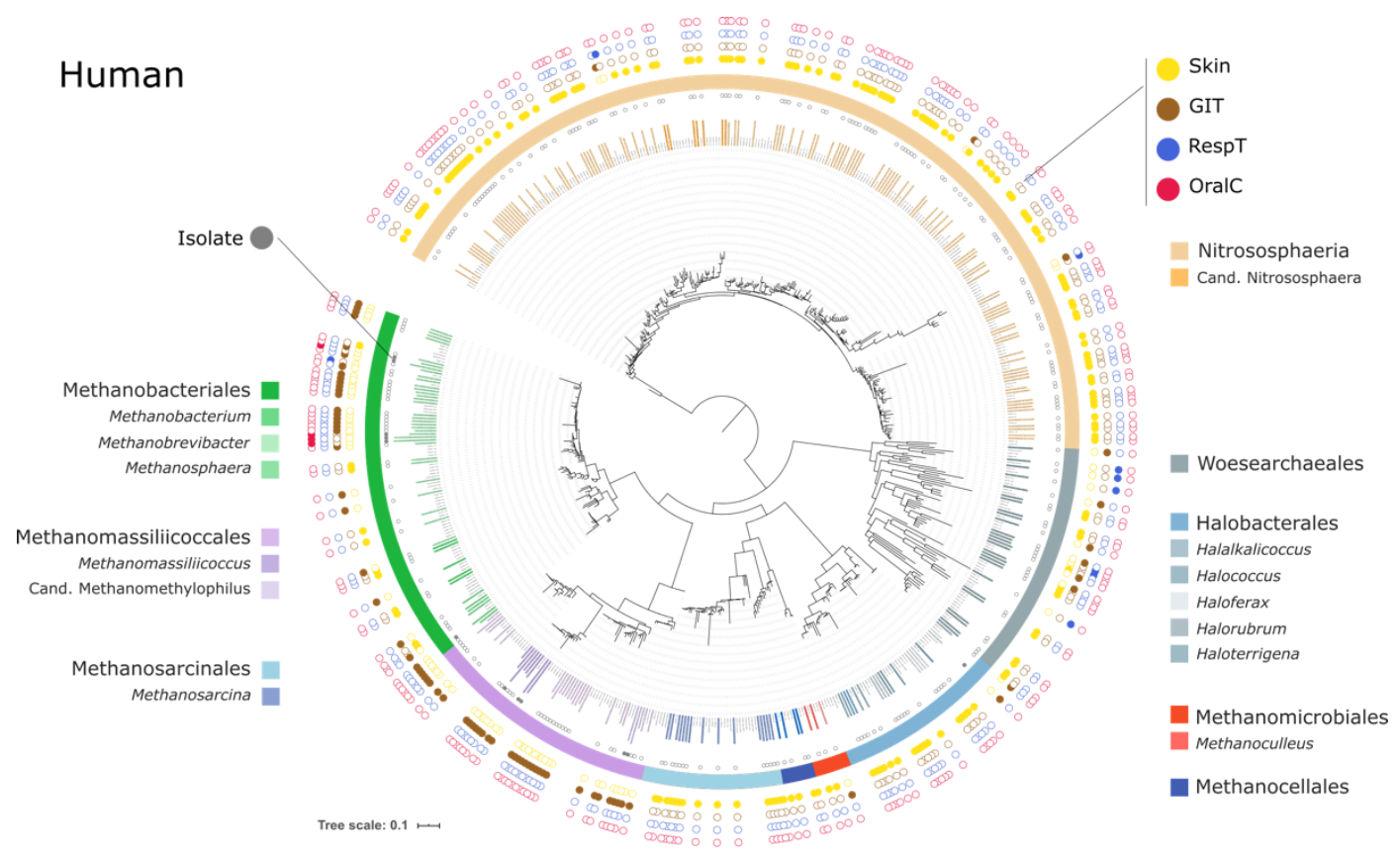

\section{Animal}

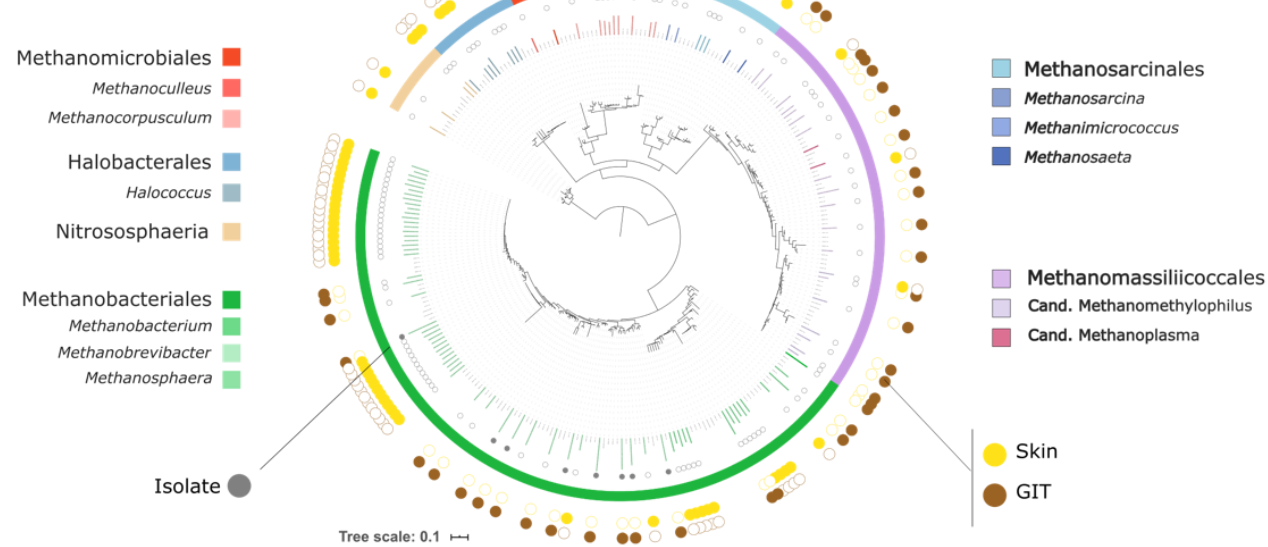

Plant

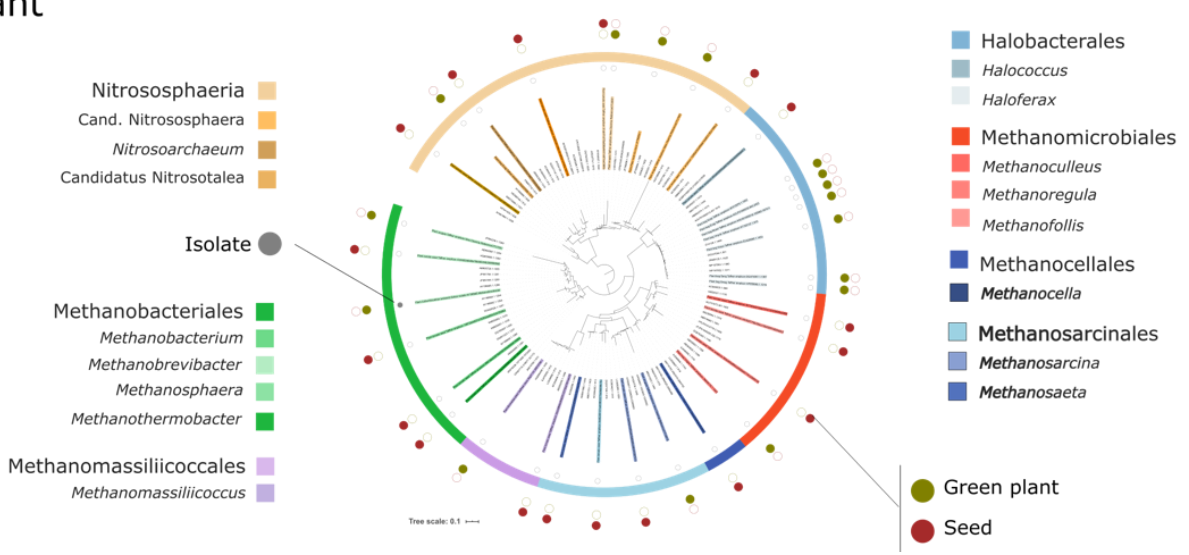

Figure 3 


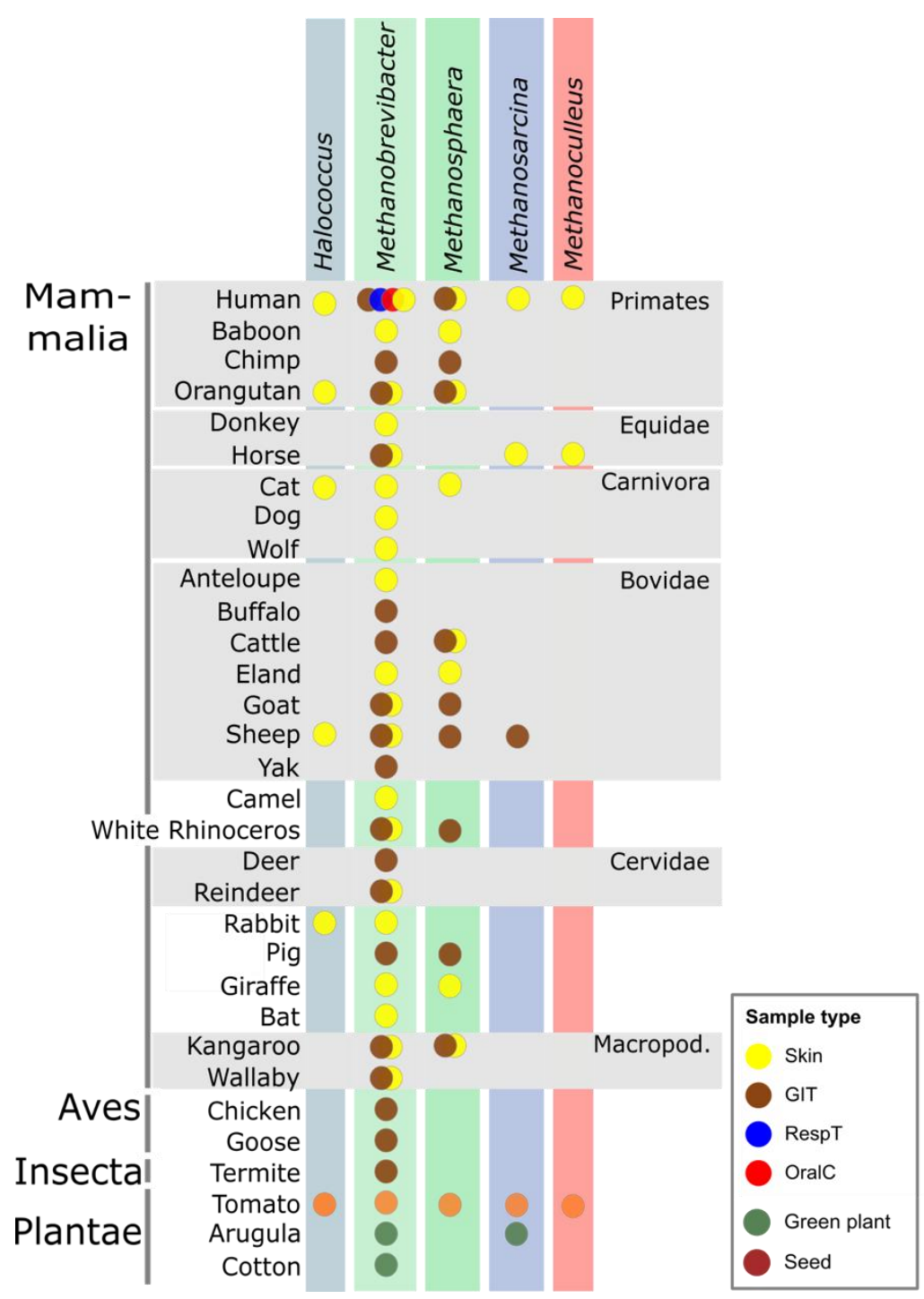

Figure 4
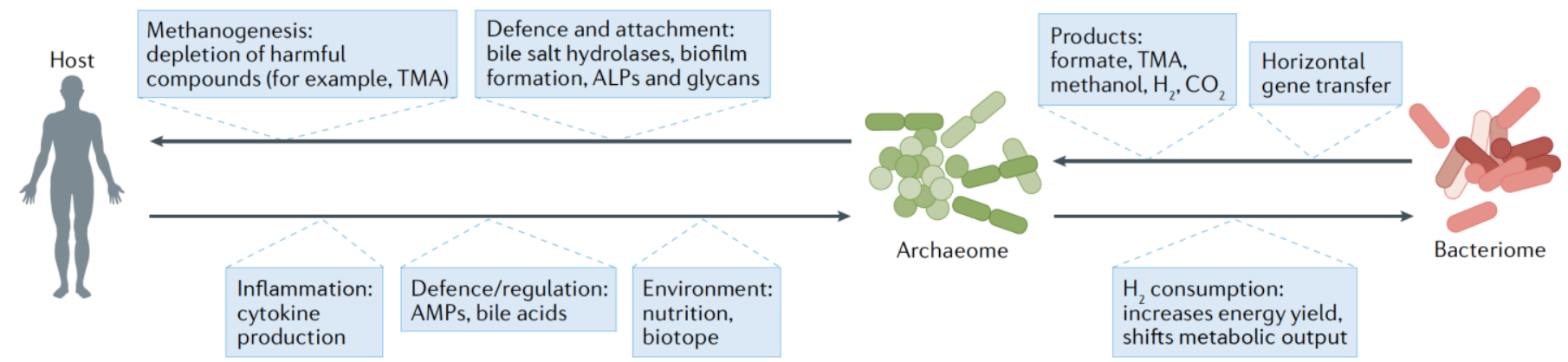

Figure 5 